\title{
Orchard Worker Localisation Relative to a Vehicle Using Radio Ranging and Trilateration
}

\author{
Stavros G. Vougioukas, Long He, Rajkishan Arikapudi \\ University of California, Davis, Department of Biological and Agricultural Engineering, \\ One Shields Avenue Davis, CA 95616-5270, USA
}

Abstract. Safe navigation of labour-aiding robots in commercial orchards will rely on accurate and continuous worker localisation. In this work, an ultra-wideband radio-based system localises a worker via trilateration of four range measurements between antennas on a vehicle and an antenna carried on the worker's belt. Performance results are presented from measurements inside 'work zones' around the vehicle, in open space and in an orchard. At walking speed in open space, when body placement allowed full line-of-sight (LOS) between belt and vehicle antennas, position estimate availability was 99.7\% and the distance root mean square error (DRMS) was $57.9 \mathrm{~cm}$. Completely blocked LOS resulted in signal outages and unacceptable performance (11.1\% availability; $819.7 \mathrm{~cm}$ DRMS). In the orchard, full-LOS performance was similar to that in open-space: $99.3 \%$ availability and $63.4 \mathrm{~cm}$ DRMS error bound. Orchard trees enabled multipath signal propagation, so blocked-LOS performance was far better than in open-space (60.2\% availability; $123.6 \mathrm{~cm}$ DRMS). Antenna motion effects were studied in open-space and orchard experiments without body interference. Motion introduced non-collocation errors (individual ranges measured at slightly different positions); DRMS error in open space and orchard were 1.6 and 2.2 times larger than respective static errors. In all experiments the $95^{\text {th }}$ percentiles of the errors were almost twice as large as the DRMS errors. Sporadic large errors and signal outages could be addressed by two belt antennas and filtering. The results indicate that radio ranging offers a practicable approach to orchard worker localisation relative to a nearby vehicle operating at slow walking speeds.

Keywords. Robots; safety; positioning; trilateration; radio sensor. 
ABSEx:

30

$31 B_{i}$ :

$32 B_{m}$ :

$33 d_{i}$ :

34 DRMS:

$35 e_{\mathrm{x}}$ :

$36 e_{\mathrm{y}}$ :

$37 e_{\mathrm{xy}}$ :

38 GPSv:

39 GPSm:

40 LOS:

$41 \quad O_{\mathrm{v}}\left(x_{\mathrm{v}}, y_{\mathrm{v}}, z_{\mathrm{v}}\right)$ :

$42 \quad O_{\mathrm{g}}\left(x_{\mathrm{g}}, y_{\mathrm{g}}, z_{\mathrm{g}}\right)$ :

43

$44{ }^{G} P$ :

$45{ }^{T} P$ :

$46{ }^{V} P_{G}$ :

$47{ }_{G}^{V} R$ :

$48{ }_{T}^{V} R$ :

$49{ }^{V} P_{T}:$

50 R95:

51

$52 \quad r_{i}$ :

$53 \quad t_{i}$ :

54 TS:

$55 \operatorname{var}\left(x_{r}\right)$ :

$56 \operatorname{var}\left(\mathrm{y}_{r}\right)$ :

$57 \quad\left(x_{\mathrm{r}}, y_{\mathrm{r}}, z_{\mathrm{r}}\right)$ :

$58 \quad\left(x_{\mathrm{i}}, y_{\mathrm{i}}, z_{\mathrm{i}}\right)$ :
Mean absolute error parallel to vehicle frame $\mathrm{x}$-axis.

Mean absolute error parallel to vehicle frame y-axis.

$i$ th vehicle radio beacon $(i=1,2,3,4)$.

Mobile radio beacon.

True distance between $B_{i}$ and $B_{m}$.

Horizontal distance root mean square position estimation error.

Position estimation error parallel to vehicle frame $\mathrm{x}$-axis.

Position estimation error parallel to vehicle frame y-axis.

Horizontal position estimation error.

Real time kinematic (RTK) GPS on vehicle.

Real time kinematic (RTK) GPS mobile; carried with mobile radio.

Line of sight.

Vehicle coordinate system.

GPSv coordinate system.

Total Station coordinate system.

GPSv coordinates in world frame.

Position expressed in TS frame.

GPSv coordinates expressed in vehicle $O_{\mathrm{v}}$ frame.

Rotation matrix from $O_{\mathrm{g}}$ to $O_{\mathrm{v}}$ frame.

Rotation matrix from $O_{\mathrm{ts}}$ to $O_{\mathrm{v}}$ frame.

Position measured by TS, expressed in vehicle $O_{\mathrm{v}}$ frame.

Radius of a circle centered at the true position, containing the position estimate with probability of $95 \%$.

Measured range between $B_{i}$ and $B_{m}$.

Time stamp of measurement $r_{i}$.

Total station.

Asymptotic variance of the non-linear least-squares estimator of $x_{r}$. Asymptotic variance of the non-linear least-squares estimator of $y_{r}$. Estimated position of mobile beacon's antenna via trilateration. ith vehicle beacon antenna coordinates. 
$\left(x_{\mathrm{m}}, y_{\mathrm{m}}, z_{\mathrm{m}}\right): \quad \quad$ Mobile beacon antenna coordinates.

$60 \quad \varepsilon_{i}:$

$61 \sigma^{2}:$

62

63

64

65

66

67

68

69

70

71

72

73

74

75

76

77
Range measurement error in $r_{i}$.

Variance of the range error, $\varepsilon_{i}$.

\section{Introduction}

Orchard operations like spraying, pruning, thinning, mowing, and especially harvesting require intensive manual labour, which contributes significantly to fruit production cost. For example, manual harvesting for apples and sweet cherries accounts for approximately $30 \%$ and $50 \%$ of production costs respectively (Bull, 2011). Meanwhile, labour availability has decreased in recent years (Hertz and Zahniser, 2013). The combination of high labour cost and labour shortage is a key driver towards the development and adoption of automation technologies that increase the productivity of orchard operations. Towards this goal, researchers have demonstrated that properly designed automated tractors, utility vehicles and labour aids can achieve productivity improvements that range from $20 \%$ up to 58\% (Moorehead et al., 2012; Bergerman et al., 2012; Ye, et al., 2013). Worker health risks, such as exposure to chemicals or ladder-related accidents can also be reduced. Research on autonomous vehicles for orchard operations has focused on problems like path planning (e.g., Linker and Blass, 2008; Bochtis et al., 2015) and navigation (e.g., Zhang et al., 1999; Barawid et al., 2007). Less attention has been paid to autonomous operation in close proximity with humans, something that is necessary for the anticipated labour efficiency benefits to be achieved in a safe manner.

Industrial robot safety has been addressed by the International Organization for Standardization (ISO) through ISO 10218, entitled "Robots and robotic devices - Safety requirements for industrial robots," which was most recently updated in 2011 (ISO, 2011). A technical specification (ISO TS 15066), entitled "Robots and robotic devices - Safety requirements for industrial robots Collaborative operation" addresses industrial robot operation in the proximity of humans and is still under development (ISO, 2014). This document provides information and guidance on how to achieve the safety standards described in ISO 10218. A key element in these standards is the ability to 
track people within a workspace and adjust robot motion according to the distance of separation between the human and robot. Although industrial and agricultural applications are not directly comparable, the ability to detect and localise nearby workers is a necessary condition for safe, "human-aware" autonomous navigation in all application areas.

Significant research has been performed in the related area of pedestrian detection and tracking in urban environments. Various active sensors such as ultrasound, radar or Lidar have been used for pedestrian detection (Gandhi and Trivedi, 2007). Extensive research on passive imaging sensors and computer vision has also been done (Dollar et al., 2012). Bellotto and $\mathrm{Hu}$ (2009) used both laser scanner and vision to detect the upper and lower parts of humans. The potential utilisation of pedestrian detection techniques for improving workforce safety in agricultural environments has been discussed by Kohanbash et al., (2012). Freitas et al. (2012) use a 3D laser scanner to detect obstacles and people moving at walking speed in front of an autonomous orchard vehicle. However, the ability of cameras and laser scanners to detect humans can be affected by environmental conditions such as low illumination, haze, fog, and rain. Also, such sensors require clear line of sight (LOS), and using them to detect a worker who is located close to trees, or who is about to enter the robot's current orchard row from a neighbouring row can be very difficult due to severe LOS occlusion from tree foliage.

The goals of this paper are to present the implementation of an ultra-wideband (UWB) radiobased system for worker localisation in orchards, and to evaluate experimentally the accuracy and availability of the system at locations within the driving reach of a moving vehicle in open space and orchard environments, with and without interference from the human's body. It is envisioned that each worker will carry a mobile radio (and its small antenna), possibly attached to a belt on his/her waist. Orchard vehicles will also carry ranging radios and the worker's position will be estimated using trilateration of the antenna-to-antenna distances between the worker and the vehicle antennas. UWB transmission is a promising radio-based technology due to its power efficiency, fine spatial 
resolution, and robust operation in harsh environments. The Federal Communications Commission (FCC) has recently allocated a spectral mask between $3.1-10.6 \mathrm{GHz}$ for UWB. Our work is motivated by similar efforts that aimed at increased reliability in harsh work environments using radio beacons carried by workers. UWB ranging can provide a complementary human detection and localisation technology whose performance is less degraded in the poor visibility condition or real complex environment that plague other sensing technologies (Chang et al., 2009). UWB radios have been proposed for human detection and localisation in harsh environments associated with construction (Khoury and Kamat, 2009; Cheng, 2011; Niemeier et al., 2014) and mining (Chehri et al., 2009; Sunderman and Waynert, 2012). Results on tracking cows in a semi-open free-stall barn using a commercial system have also been reported (Porto et al., 2014).

This work investigates UWB operation in agricultural environments, because it is well known that the water contained in vegetation attenuates radio signals, and that signal reflection on the ground and scattering at tree branches introduce multipath effects that reduce accuracy (Vougioukas et al., 2013). Also, the presence of human bodies in the radio wave transmission path has been shown to affect ranging accuracy or even block transmission in indoors applications (Welch et al., 2002) and other confined spaces like mining (Mabrouk et al., 2012). Since the mobile antenna is to be carried by workers, human body interference in free/open space and in an orchard is examined.

\section{Materials and Methods}

An experimental position estimation system was developed to localise mobile UWB beacons that are to be carried by orchard workers. The mobile beacon antenna position coordinates were computed (with respect to a vehicle) by trilateration, using the measured ranges between the mobile beacon antenna and four beacon antennas on an autonomous vehicle. It is well known (Manolakis 1996) that three ranges suffice for trilateration on the plane. However, the LOS between a vehicle beacon and worker antennas will often be blocked in an orchard by vegetation or people. Furthermore, it has been shown (Zhou, 2011) that the estimation error and the uncertainty with four reference points tends to 
135 be lower than that with three reference points when the object is located farther away; hence, four

136 beacons were used for increased accuracy and redundancy. The accuracy and availability of the 137 positioning system were assessed around the vehicle under static and dynamic walking-speed 138 conditions, in open space and in an orchard.

139

140

141

142

143

144

145

146

147

148

149

150

151

152

153

154

\subsection{Experimental materials}

As shown in Fig. 1, the experimental system consists of several components. A computer-controlled electric utility vehicle (Sensible Machines, Pittsburgh, PA, USA) was used as a mobile platform that carries radio beacons. Four ranging radio receiver-transmitter units (P400 RCM, Time Domain, Huntsville, AL, USA) mounted on wooden poles on the vehicle were used as vehicle beacons. One P400 RCM radio carried by a person was used as a mobile beacon. One RTK GPS (AgGPS 542, Trimble, Sunnyvale, CA, USA) on the vehicle (referred to as "GPSv") and an identical mobile RTK GPS (referred to as "GPSm") carried by a person were used to measure vehicle and antenna locations. A serial to Wi-Fi adaptor (WA-232B, U.S. Converters LLC, Sedona, AZ, USA) was used to transmit the mobile GPS data to the data-logging computer. A Total Station (CST-305R, CST/berger, Watseka, IL) was used to measure true mobile antenna positions; it will be referred to as "TS". The TS has 5-second angle accuracy at a sighting distance of $100 \mathrm{~m}$, which is equivalent to an accuracy of $2.5 \mathrm{~mm}$ in distance measurement. A portable aluminium frame was constructed to carry the mobile beacon and the GPSm at various locations. The mobile beacon's antenna was placed above the GPSm at a height equal to $0.9 \mathrm{~m}$ (human's waist level), in a vertical orientation aligned to the vertical axis of the GPSm reference frame. 


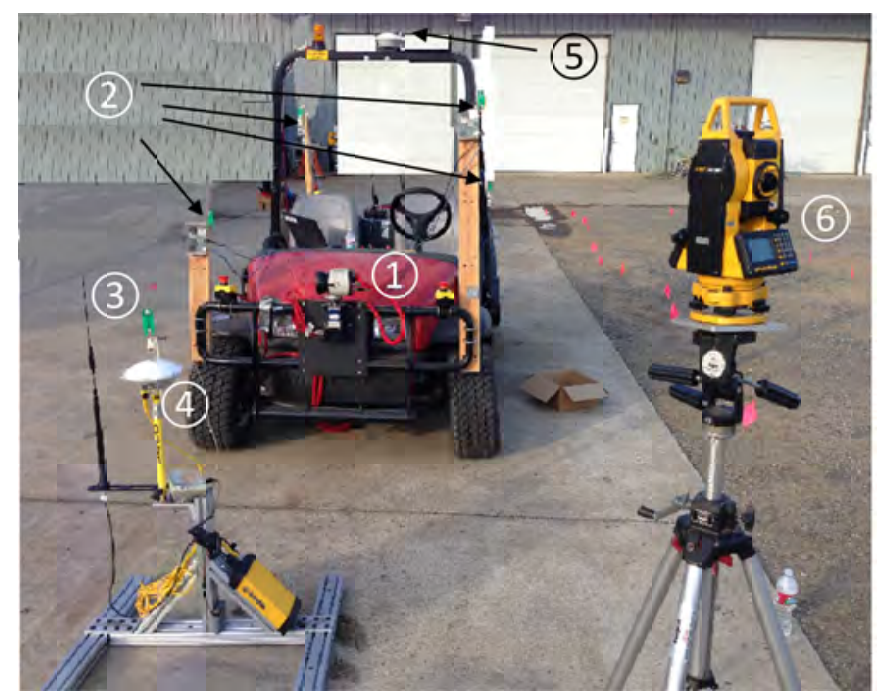

Fig. 1 - The UWB localisation system consists of: 1) utility vehicle, 2) four vehicle radio beacons, 3) mobile radio beacon, 4) mobile RTK GPS, 5) vehicle RTK GPS, and 6) Total Station. RCM antennas (Fig. 2). The units operate in the band of $3.1 \mathrm{GHz}$ to $5.3 \mathrm{GHz}$ with a centre frequency at $4.3 \mathrm{GHz}$. In a static scenario (antennas not moving) with a clear LOS, the specifications for the range measurement precision and accuracy are $2.3 \mathrm{~cm}$ and $2.1 \mathrm{~cm}$ respectively. Each radio unit on the vehicle (referred to as "beacon") reports its distance from the mobile radio every $20 \mathrm{~ms}$ within a maximum range of $125 \mathrm{~m}$.

168

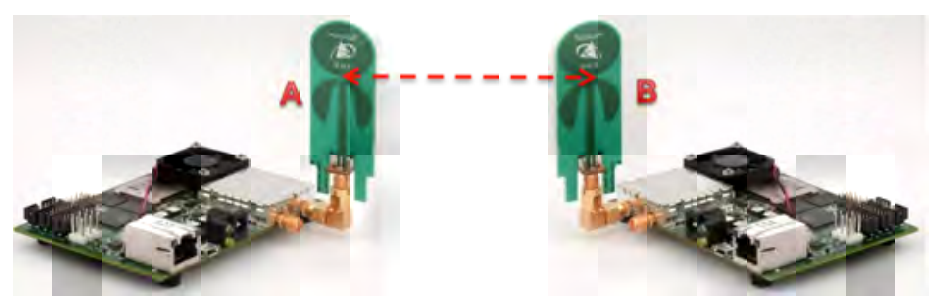

Fig. 2 - Radio unit A measures and reports the distance (red dotted line) between the centre points of antennas $\mathrm{A}$ and $\mathrm{B}$. 
171 In our experiments each range measurement was calculated as the median value of five consecutive

172 range measurements, in order to reject outliers. Hence, a new filtered range update was available for 173 each beacon approximately every $100 \mathrm{~ms}$. The response times of the beacons would vary slightly and 174 therefore the update rate for trilaterated position estimates based on the range data was approximately $175400 \mathrm{~ms}$, i.e., $2.5 \mathrm{~Hz}$. The GPS units were polled every $500 \mathrm{~ms}$, i.e., at $2 \mathrm{~Hz}$. Each beacon returns a 176 value of zero as range measurement if it does not get a response from the mobile radio (e.g., due to 177 obstacles in the transmission path) within the specified time window of $20 \mathrm{~ms}$.

178 In this study the position of the mobile radio antenna was estimated via trilateration using the radio 179 beacons, and was measured directly using the GPSm or the TS. Positions measured by the TS were 180 considered to be the most accurate ones. However, the TS requires a stationary target and 181 unobstructed LOS from the target. Therefore, it could only be used for static tests in open space and in 182 the orchard when LOS was available. For dynamic tests and static tests without LOS the GPSm was 183 used as ground truth.

184 A multi-threaded data acquisition program was developed in Visual $\mathrm{C}++$ to poll data from different 185 sources without response delays. One thread would poll sequentially the four vehicle beacons to 186 collect range data; a second thread would retrieve and parse the strings provided by the GPS receivers 187 and a third thread would log the data.

\subsection{System Coordinate frames}

The data collected from different devices had their own coordinate systems. To process the data, each individual coordinate system was transformed into a single common primary coordinate system. The GPS and the TS coordinate systems were transformed into the vehicle coordinate system. The 193 coordinate systems of the vehicle, $O_{\mathrm{v}}\left(x_{\mathrm{v}}, y_{\mathrm{v}}, z_{\mathrm{v}}\right)$, the TS, $O_{\mathrm{ts}}\left(x_{\mathrm{ts}}, y_{\mathrm{ts}}, z_{\mathrm{ts}}\right)$, and the GPSv $O_{\mathrm{g}}\left(x_{\mathrm{g}}, y_{\mathrm{g}}, z_{\mathrm{g}}\right)$ are 194 shown in Fig. 3. The origin of vehicle coordinate system was placed at the same point as the origin of 
195

196

197

198

199

200

201

202

203

204

205

206

207

the GPSv coordinate system. The geometric centreline (viewed from above the vehicle) is an imaginary line drawn from the centre of the rear axle to the centre of the front axle. The vehicle centreline was used to define the $\mathrm{x}$-axis of the vehicle coordinate system. The angle $\theta$ between the $y$ axes of the GPSv and the vehicle coordinate systems was measured using the two RTK GPS units. The y-axis of the GPS coordinate system was set to the North direction, and the x-axis to the East direction. The orientations of the $x$ - and $y$-axis were the same for the vehicle and the TS coordinate systems, albeit with different origins. The z-axes of the three coordinate systems were vertical upwards from the ground plane.

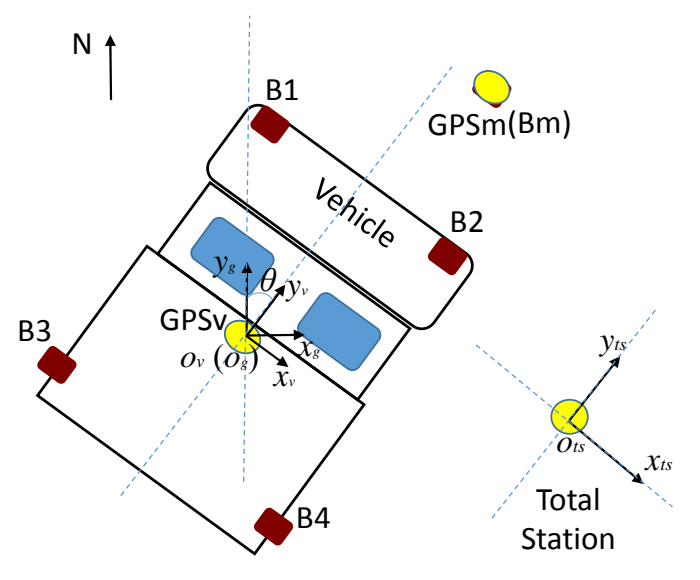

Fig. 3 - Coordinate frames of the vehicle, radio beacons, GPSv, GPSm, and TS.

The data measured in the GPSv coordinate frame were expressed in the vehicle frame as follows:

$$
{ }^{V} P_{G}={ }_{G}^{V} R \cdot{ }^{G} P=\left[\begin{array}{ccc}
\cos \theta & -\sin \theta & 0 \\
\sin \theta & \cos \theta & 0 \\
0 & 0 & 1
\end{array}\right]{ }^{G} P
$$
where, ${ }^{V} P_{G}$ is the coordinates vector of the position calculated by the GPS expressed in the vehicle

209 frame, ${ }_{G}^{V} R$ is the rotation matrix between the GPS and the vehicle frames, ${ }^{G} P$ is the coordinates 210 vector of points in the GPS frame, and ${ }^{V} P_{G 0}$ is the translation vector between the GPSv and vehicle 211 coordinate frames. Similarly, the positions measured from the TS can be expressed in the vehicle 


$$
{ }^{V} P={ }_{T}^{V} R \cdot{ }^{T} P+{ }^{V} P_{T 0}=\left[\begin{array}{ccc}
1 & 0 & 0 \\
0 & 1 & 0 \\
0 & 0 & 1
\end{array}\right]{ }^{T} P+\left[\begin{array}{c}
d_{x} \\
d_{y} \\
0
\end{array}\right]
$$

214 where, ${ }^{V} P_{T}$ is the coordinates vector of the position calculated by the TS in the vehicle frame, ${ }_{T}^{V} R$ is

215 the rotation matrix between the TS and vehicle frames, ${ }^{T} P$ is the coordinates vector of points in the 216 TS frame, ${ }^{V} P_{T 0}$ is the translation matrix between the TS and vehicle frames. Variables $d_{x}$ and $d_{y}$ 217 represent the distances of two origins with respect to $x$ and $y$ axes of the vehicle coordinate system, 218 which were measured using the TS at the beginning of each experiment.

\subsection{Trilateration method and performance metrics}

220 The antenna coordinates for vehicle beacon $B_{i}$ be $\left(x_{i}, y_{\mathrm{i}}, z_{\mathrm{i}}\right)(i=1,2,3,4)$, and the true antenna 221 coordinates of the mobile beacon $B_{m}$ be $\left(x_{\mathrm{m}}, y_{\mathrm{m}}, z_{\mathrm{m}}\right)$, are all expressed in the vehicle reference frame 222 (Fig. 4). The vehicle beacon coordinates were: $B_{1}=(-0.625,1.348,1.648) ; B_{2}(0.623,1.326,1.185)$; $B_{3}=(-0.675,-1.252,1.172) ; B_{4}=(0.691,-1.282,1.577)$. The positions were selected to minimise the expected mean square error in the mobile beacon position around the vehicle (He et al., 2014). The corresponding measured ranges from the vehicle beacons to the mobile beacon are $r_{1}, r_{2}, r_{3}$, and $r_{4}$ respectively.

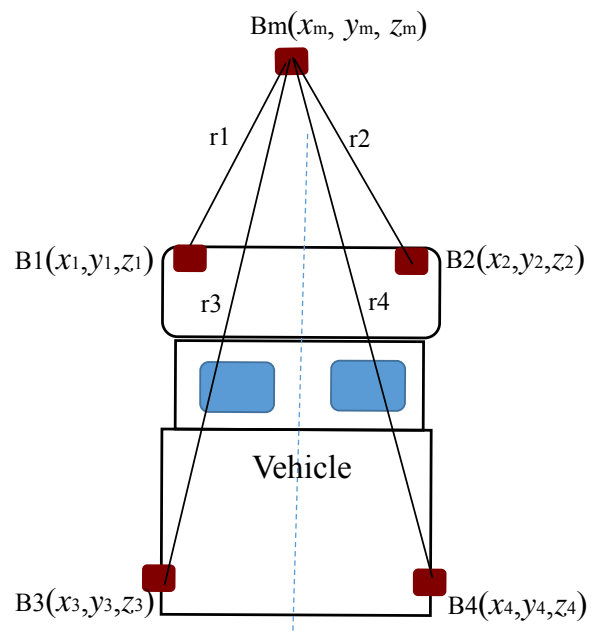


Fig. 4 - The illustration of four ranges from vehicle beacons to the mobile beacon.

The true distance $d_{\mathrm{i}}$ between the ith vehicle beacon and the mobile beacons is:

$$
d_{i}=\left(\left(x_{m}-x_{i}\right)^{2}+\left(y_{m}-y_{i}\right)^{2}+\left(z_{m}-z_{i}\right)^{2}\right)^{(1 / 2)}
$$

The measured range $r_{i}$ will contain some error $\varepsilon_{i}$, i.e.:

$$
r_{i}=d_{i}+\varepsilon_{i}
$$

Trilateration is used to estimate the coordinates of the mobile beacon based on the four measured ranges. In the presence of range errors and absence of any prior information on the error statistics the non-linear least-squares estimator is the optimal position estimator (Navidi et al., 1998). Hence the trilateration equation is:

$$
\left(x_{r}, y_{r}, z_{r}\right)=\underset{x, y, z}{\arg \min } \sum_{i=1}^{4}\left(r_{i}-d_{i}\right)^{2}
$$

One important observation is that the range measurements $r_{1}, r_{2}, r_{3}, r_{4}$ in Eq. (5) are available at times $t_{1}, t_{2}, t_{3}, t_{4}$ that are spaced $100 \mathrm{~ms}$ apart. In dynamic situations where the worker (and antenna) is moving, these ranges do not correspond to a unique worker position, but rather to four distinct positions that at walking speeds are very close to each other. Therefore, Eq. (5) will result in an estimate that contains additional error, which is independent of the range error $\varepsilon_{i}$.

To evaluate the accuracy of the developed UWB localisation system, the trilaterated positions $\left(x_{r}, y_{r}, z_{r}\right)$ were compared to the ground truth $\left(x_{m}, y_{m}, z_{m}\right)$ that was measured using the TS or GPSm. The horizontal error in the $x y$ plane, $e_{x y}$ is:

$$
e_{x y}=\sqrt{\left(x_{r}-x_{m}\right)^{2}+\left(y_{r}-y_{m}\right)^{2}}
$$

The error parallel to the vehicle's centreline (y-axis), $e_{\mathrm{y}}$, and the error perpendicular to the vehicle centreline, $e_{\mathrm{x}}$ are: 


$$
e_{x}=x_{r}-x_{m}, e_{y}=y_{r}-y_{m}
$$

251 Given a set of $N$ measurements, the system's accuracy was characterised using a number of metrics.

252 The first accuracy metric is the horizontal distance root mean square error (DRMS):

$$
D R M S=\sqrt{\frac{1}{N} \sum_{i=1}^{N}\left(e_{x i}^{2}+e_{y i}^{2}\right)}
$$

In GPS literature the 2DRMS (twice the DRMS) is used extensively. The advantage of this metric is that it is a radial error, i.e., it is invariant to the rotation of the axes used to describe the error. However, in an orchard setting where a vehicle moves along rows with a well-defined motion axis that cannot change instantly, it is important to distinguish between errors along the motion - and tree row - axis, and errors perpendicular to it. Therefore, two additional metrics were computed, i.e., the average absolute errors in the $x$ and $y$ directions:

$$
A B S E x=\frac{1}{N} \sum_{i=1}^{N}\left|e_{x i}\right|, A B S E y=\frac{1}{N} \sum_{i=1}^{N}\left|e_{y i}\right|
$$

Two disadvantages of the above metrics are that isolated large outlier errors have a large effect on them, and that the metrics are not tied to a specific probability level. Therefore, the $95^{\text {th }}$ percentile or R95 error metric is also computed based on $e_{x y}$. This metric is equal to the radius of a circle centred at the true position, containing the position estimate with probability of $95 \%$. Finally, the (vertical) errors along the $z$-axis were ignored in this work. In addition to position accuracy metrics, position availability was used to quantify the 267 performance of the proposed localization method. Each set of four successive range requests by the 268 vehicle beacons result in a quadruple $\left(r_{1}, r_{2}, r_{3}, r_{4}\right)$. Although in theory a 2D position can be derived 269 from three ranges, preliminary experiments showed that when one range measurement was equal to zero (no response) other ranges were often very noisy and unreliable, and the estimated position 271 contained very large errors. Therefore, in our experiments a range quadruple was considered 'valid' 272 only if it contained no zeros; then, its corresponding position estimate was considered 'available'. So, 
273

274 275 276

277 278 279 280 281

the position availability in a set of measurements was calculated as the ratio of the 'available' over all the requested position estimates.

All post-processing calculations were performed using Matlab R2013a (MathWorks, Natick, MA, USA), and the trilateration equation was solved using the fminsearch() Matlab optimisation function. Regarding the error calculations, it is important to notice that in dynamic situations where the worker carrying the antenna is moving, successive beacon ranges are spaced apart in time and cannot be synchronised with GPSm measurements. All errors were calculated under the simplifying assumption that the estimated worker position $\left(x_{r}, y_{r}\right)$ has the same timestamp $t_{4}$ as the last range measurement $\hat{r}_{4}$, and by selecting as ground truth the GPSm position whose timestamp was closest (within $\pm 50 \mathrm{~ms}$ ) to $t_{4}$. In a real-time scenario this would require instantaneous calculation of Eq. (5).

\subsection{Experimental Design}

Autonomous vehicles in orchards are expected to travel inside orchard rows, in headlands and in open space areas, such as farm roads. The vehicles are typically non-holonomic, with non-zero turning radius, and hence cannot move sideways or make very sharp turns. The accuracy and availability of the developed UWB radio localisation system were evaluated inside suitable 'work zones' in open space and in an orchard.

\subsubsection{Static tests in open space}

For the open space static tests without any human body interfering in the antennas' LOS, the work zone was defined as a $24 \mathrm{~m} \times 8 \mathrm{~m}$ area with the vehicle at its centre; a rectangular grid of cell size $2 \mathrm{~m}$

$\times 1 \mathrm{~m}$ was used to sample this area uniformly (Fig. 5). The dimensions of the measurement area were selected taking into account the locations where orchard workers may be present within the driving reach of a moving vehicle in typical orchard configurations ( $4 \mathrm{~m}$ wide inter-row spacing). The line 
297 defined by the vehicle's geometric centreline is labelled "1". The lines labelled " 2 " and " 3 " are two 298 and four metres away from the centreline of the vehicle at its left and right sides, and are parallel to it.

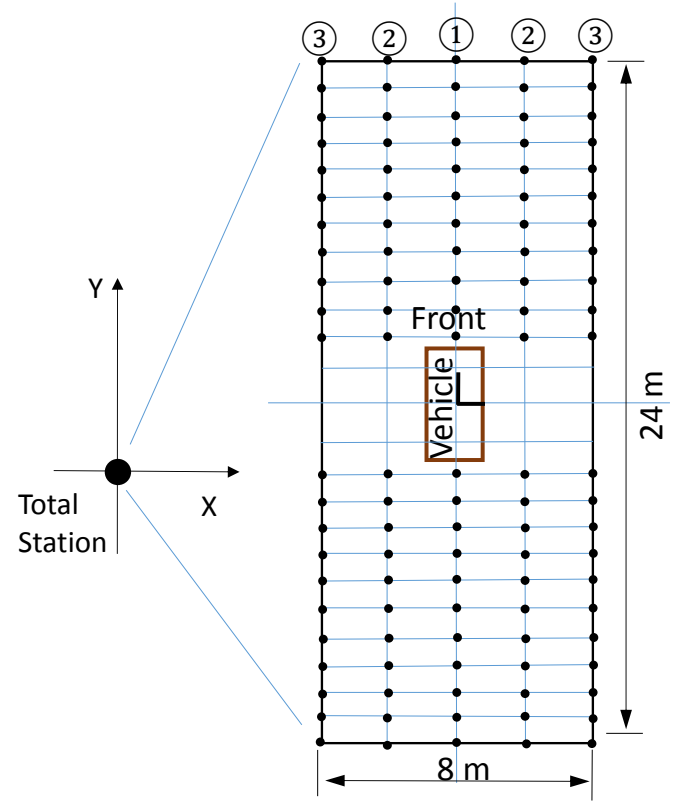

299

300

301

302

303

304

305

306

307

308

309

310

311

Fig. 5 - Static test design in open space; black dots represent the locations of the static test points.

The localisation accuracy of the system was evaluated by moving the portable frame with the mobile beacon and GPSm on the nodes of the grid (black dots in Fig. 5), and by measuring the antenna position with the radio system, the GPS and the TS. Two hundred sets of four range measurements were taken at each node.

\subsubsection{Static tests in an orchard}

Experiments were conducted in an orchard managed by the University of California - Davis. The orchard contained central-leader Bartlett pear trees that were approximately $4 \mathrm{~m}$ high. The average height of the trunk from the ground to the first scaffolds, or major limbs, was $0.6 \mathrm{~m}$ (Fig. 6). The row spacing in the orchard was $3.6 \mathrm{~m}$. 


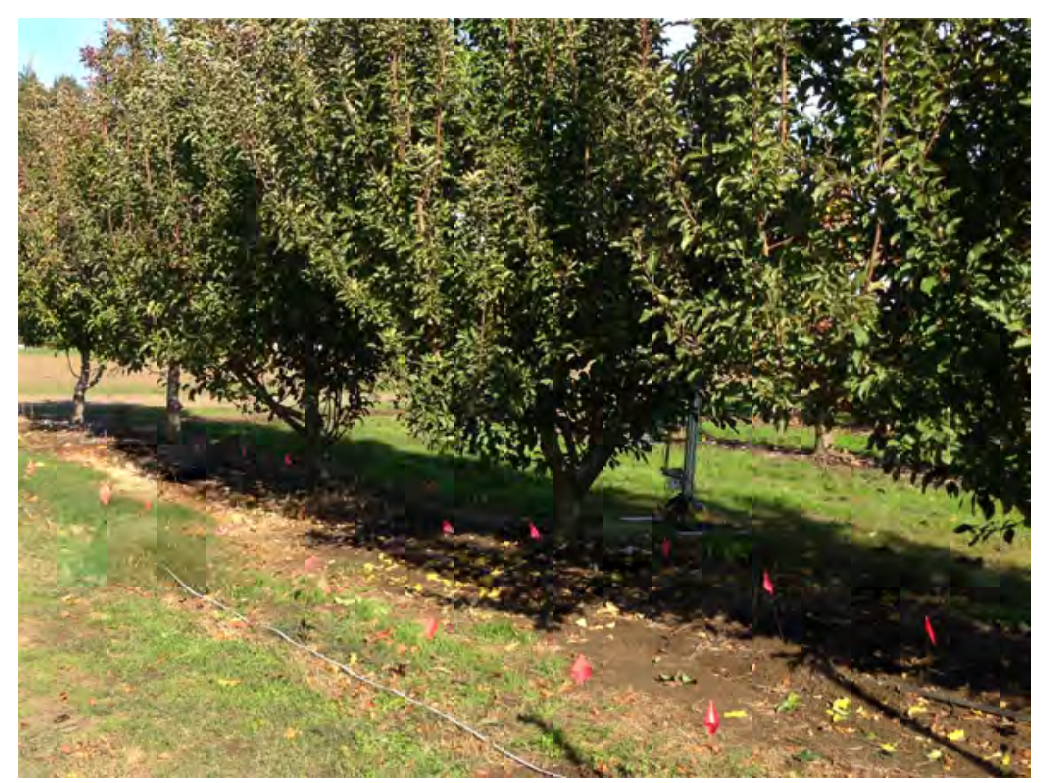

315 Fig. 6 - Image from the pear tree orchard used for localisation experiments.

335 According to the experimental design, the work zone was defined as a $24 \mathrm{~m} \times 3.6 \mathrm{~m}$ area with the 336 vehicle at its right side, and the vehicle was placed to the right of a row at the edge of the orchard and 337 parallel to it (Fig. 7). The edge row was selected in order to provide enough distance between the TS 338 and the measurement points, so that the incidence angle of the laser beam on the antenna surface is 339 large (close to $90^{\circ}$ ) at all points. A large incidence angle translates to small laser beam trace on the 340 antenna (better accuracy) and to higher intensity for the reflected beam, which is required for valid 341 measurements. Orchard tests took place in late May and early June 2015 and the trees had full foliage; 342 fruit size was very small. During 'static' experiments the mobile radio antenna was held stationary 343 during all range measurements. During 'dynamic' experiments the antenna was moving relative to the 344 vehicle at walking speed. 


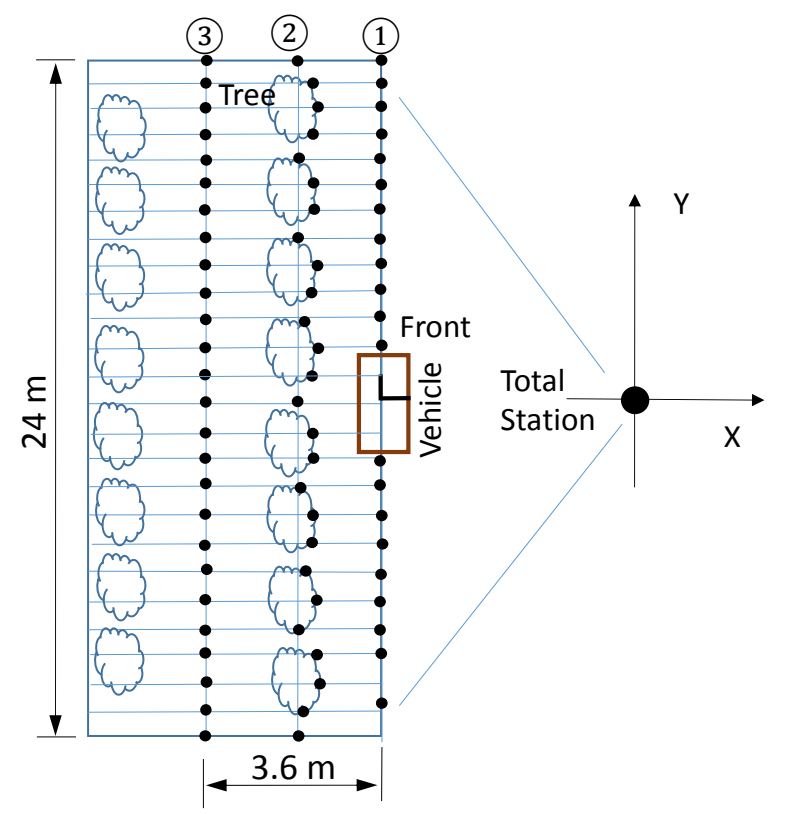

325

326

327 328

Fig. 7 - Static test design in orchard environment; black dots represent the locations of the static test points.

The position error was assessed at three sets of points located on - or close to - three lines parallel to the TS's y-axis (Fig. 7; tree sketches are not drawn in scale). All points were spaced at approximately $1.0 \mathrm{~m}$ along the TS y-axis. Line No. 1 was defined by the geometric centreline of the vehicle. Line No. 2 was defined by the tree trunks; however, the actual measurement positions were on points along the peripheries of the canopies where GPS signal was available. Line No. 3 was the middle-row line between two consecutive tree rows to the left of the vehicle. Points on this line correspond to possible locations of orchard workers working and walking in proximity to the vehicle without clear LOS from the vehicle. Detecting and localising workers on points on line No. 3 would be challenging for any detection system relying on optics (camera, laser) because worker visibility is severely restricted by tree trunks and foliage. The true positions for the points on line No. 3 were to be measured using the GPSm only, because tree foliage blocked their line of sight from the TS. Two hundred sets of four range measurements were taken at each point on each line. 


\subsubsection{Dynamic tests in open space and orchard}

343 In order to evaluate the accuracy of the developed radio localisation system when the mobile and

344 beacon antennas move with respect to each other, dynamic tests were designed in open space and in 345 an orchard; no human bodies interfered in the antennas' LOS during these tests. During these tests the 346 antenna was moved relative to the vehicle at slow walking speed, close to $1 \mathrm{~km} \mathrm{~h}^{-1}$. A slow speed was 347 selected because this paper focuses on autonomous labour-aid vehicles, where the relative speeds 348 between workers and vehicles are small (workers may pick fruits, prune, perform flower-thinning, 349 etc.).

350 The dynamic tests in open space were designed to take place on the paths defined by the lines labelled 351 "1", "2" and "3", as shown in Fig. 8a; these were defined in the same manner as the lines in the static 352 tests (Fig. 5). Similarly, the orchard dynamic tests were designed for the paths defined in the static 353 orchard tests. As shown in Fig. 8b, one path was along the centreline of the vehicle (line 1), another 354 path was along the peripheries of the tree canopies (path 2) and a third path was at the middle of the 355 neighbouring row (line 3). Each path was traversed ten times.

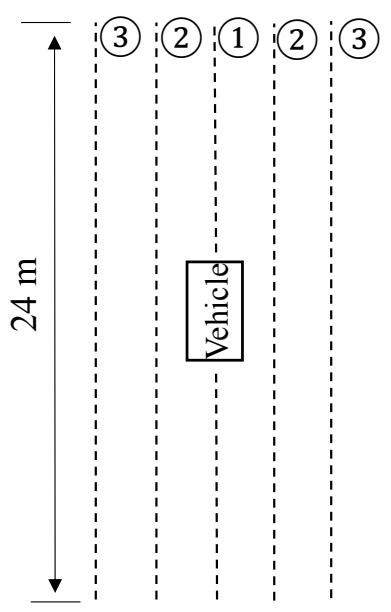

a)

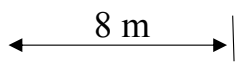

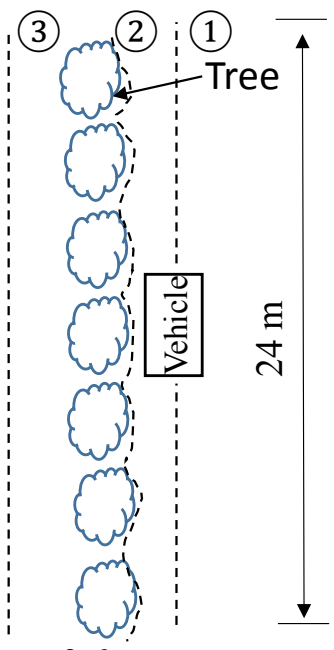

b)

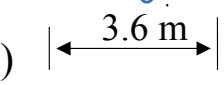

357 Fig. 8 - Dynamic test paths in: (a) open space, and (b) in an orchard. 
As explained in the introduction, it is envisaged that each worker would carry a mobile UWB

radio and an antenna that is attached to a belt on his/her waist. It has been shown experimentally (Welch et al., 2002) that the human body causes severe signal attenuation when it stands in the LOS between an antenna attached to the body and an antenna far from it. Therefore, in a light multipath environment where the signals exchanged between the wearable antenna and the receiving beacons travel directly and not via reflections, the power received from the wearable antenna depends strongly on the person's orientation with respect to the receiving beacon (degree of LOS blocking). This dependence is greatly reduced in dense multipath environments where signals make it to the receiving beacon via reflections (Welch et al., 2002). Localisation accuracy of a wearable antenna in an indoor light multipath environment has been recently explored experimentally at close range (inside a $1.5 \mathrm{mx}$ $1.5 \mathrm{~m}$ area) and shown to depend on body angle (Bharadwaj, 2015). UWB-based localisation availability and accuracy at large distances and in the presence of foliage has not been reported in the literature.

In order to evaluate human body interference in our proposed localization scenario where the beacons are on a vehicle, the antenna was placed on a belt attached to the waist of a walking person, and two sets of experiments were performed: one in open space and one in the orchard.

In the open-space set of experiments, the vehicle was positioned at a location where there were no nearby objects or trees that could reflect the signals between the wearable antenna and the vehicle beacons. The person carrying the UWB radio and antenna walked on lines 1, 2 and 3 as shown in Fig. 8a. Since we know that body orientation is important for LOS communication between antennas, and given that in real situations a worker's orientation with respect to the vehicle antennas will be random, two experiments were performed in open space. In the 'best case' experiment the person walked making sure that the wearable antenna always faced the vehicle beacons and had unobstructed LOS with all of them. In the 'worst case' experiment the walking person's body always blocked completely the LOS between the wearable antenna and each of the vehicle beacons. From now on, these 
experiments will be referred to as 'full-LOS' and 'no-LOS' experiments respectively. The accuracies

386

387 and availabilities calculated from these experiments offer bounds of performance for real-life orchard activities. Each experiment was replicated ten times.

In the orchard set of experiments the person walked along the lines 1, 2 and 3 shown in Fig. 8b. The first orchard experiment was a full-LOS one, and the second experiment was a no-LOS one, as these were described in the previous paragraph; each experiment was replicated ten times.

\section{Results and discussion}

\subsection{Open-space results}

\subsubsection{Static tests in open space}

The antenna positions at the grid points were measured using the TS and GPSm, and were estimated using UWB ranging and trilateration; these positions are shown in Fig. 9 for a specific test. The TS measurements are not visible at many locations because they lie below the dots representing the GPSm measurements. The DRMS and R95 metrics for the distance between the TS and GPSm measurements were $1.8 \mathrm{~cm}$ and $3.2 \mathrm{~cm}$ respectively, whereas the maximum distance was $4.0 \mathrm{~cm}$. Furthermore, a two-sample Kolmogorov-Smirnov test verified that the error data with TS and GPSm as ground truth have the same distribution at the 5\% significance level. Hence, for the purposes of this work the TS and GPSm provide adequate accuracy and can be used interchangeably as ground truth in open-space tests. 


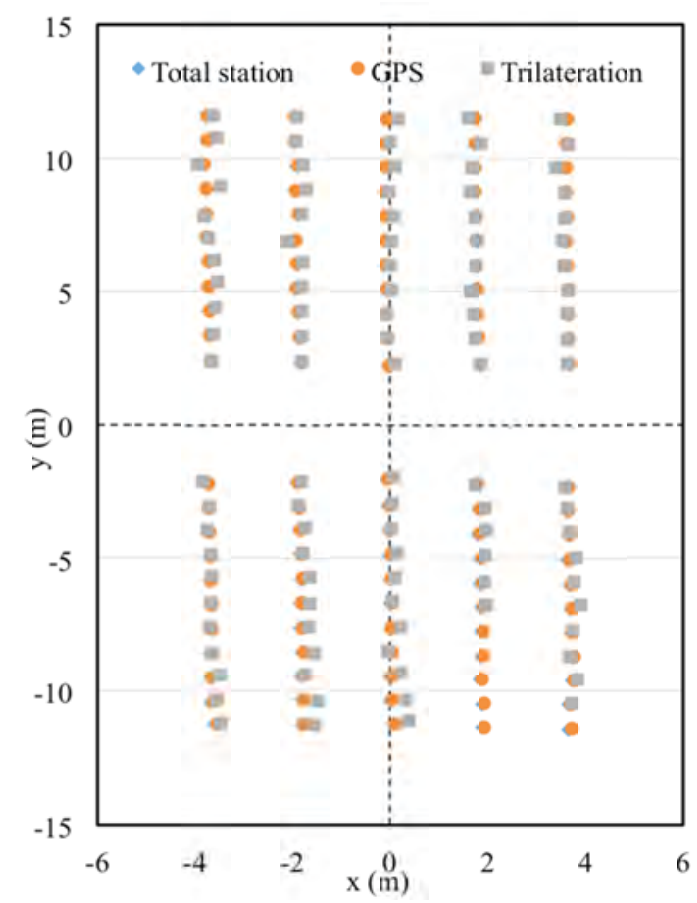

405

410

411

412

409

412

413

412

413

414

\begin{tabular}{|lcccc|}
\hline & DRMS & ABSEx \pm std & ABSEy \pm std & R95 \\
Line 1 & 17.7 & $13.6 \pm 8.8$ & $5.5 \pm 4.8$ & 33.9 \\
Line 2 & 14.5 & $10.9 \pm 7.8$ & $4.7 \pm 3.5$ & 26.7 \\
Line 3 & 13.3 & $8.1 \pm 6.8$ & $6.5 \pm 4.7$ & 24.3 \\
Front-all & 12.4 & $9.2 \pm 6.5$ & $3.8 \pm 3.7$ & 24.1 \\
Rear-all & 17.0 & $11.6 \pm 9.0$ & $7.7 \pm 4.2$ & 33.6 \\
All points & 14.8 & $10.3 \pm 7.8$ & $5.6 \pm 4.4$ & 27.2 \\
\hline
\end{tabular}
represent the average trilateration estimate for each position. respectively.

Table 1 - Open-space static accuracy metrics using TS as ground truth (units in $\mathrm{cm}$ ).

Fig. 9 - Open-space static test: positions measured with TS and GPSm and estimated via trilateration in a specific test. Diamonds represent TS measurements; dots represent GPSm measurements; squares

The position errors are illustrated in Table 1 and Table 2, using TS and GPSm as ground truth

Table 2 - Open-space static accuracy metrics using GPSm as ground truth (units in $\mathrm{cm}$ ). 


\begin{tabular}{|lcccc|}
\hline Line 1 & 17.1 & $13.3 \pm 8.1$ & $2.3 \pm 4.9$ & 30.7 \\
Line 2 & 13.8 & $10.5 \pm 7.1$ & $3.5 \pm 3.4$ & 24.8 \\
Line 3 & 13.4 & $8.0 \pm 7.1$ & $5.0 \pm 4.7$ & 25.6 \\
Front-all & 13.0 & $9.7 \pm 6.9$ & $3.9 \pm 3.7$ & 25.5 \\
Rear-all & 15.9 & $10.4 \pm 8.2$ & $7.9 \pm 4.1$ & 30.6 \\
All points & 14.4 & $10.0 \pm 7.5$ & $5.8 \pm 4.4$ & 25.7 \\
\hline
\end{tabular}

416

417

418

419

420

421

422

423

424

425

426

427

428

429

430

431

432

433

434

Both tables show that the ABSEx errors are significantly larger than the ABSEy errors along each line. A two-sample Kolmogorov-Smirnov test was applied on the ABSEx and ABSEy errors and the hypothesis that these errors come from identical distributions was rejected ( $5 \%$ significance).

As will be explained next, the explanation for this lies in two factors: a) the geometrical configuration of the vehicle beacons and its effect on precision along the orthogonal axes; and b) the geometry of the work zone. It is known (Navidi et al., 1998) that for orthogonally placed beacons (like the $\mathrm{B}_{\mathrm{i}} \mathrm{s}$ ) the asymptotic variances of the non-linear least-squares estimator $\left(x_{r}, y_{r}\right)$ for the trilaterated coordinates in the $x$ and $y$-axes are:

$$
\operatorname{var}\left(x_{r}\right)=\frac{\sigma^{2}}{\sum_{i=1}^{4} \frac{\left(x_{m}-x_{i}\right)^{2}}{d_{i}^{2}}}, \operatorname{var}\left(y_{r}\right)=\frac{\sigma^{2}}{\sum_{i=1}^{4} \frac{\left(y_{m}-y_{i}\right)^{2}}{d_{i}^{2}}}
$$

where $\sigma^{2}$ is the variance of the range error. The mean absolute errors ABSEx and ABSEy are proportional to $\operatorname{var}\left(x_{r}\right)$ and $\operatorname{var}\left(y_{r}\right)$ respectively. The reason is that the errors $e_{x}$ and $e_{y}$ follow normal distributions (verified via a one-sample Kolmogorov-Smirnov test). The absolute values $\left|e_{x}\right|$ and $\left|e_{y}\right|$ follow folded normal distributions, and the mean of the folded normal distribution is proportional to the variance of the original normal distribution (Leone, Nottingham \& Nelson, 1961). Theoretical evaluation of Eq. (10) was performed on a $24 \times 24 \mathrm{~m}$ region centred at the vehicle. The value of each pixel in Fig. 10 shows whether the ratio ABSEx/ABSEy is greater, less or equal to one. The figure reveals that accuracy along the y-axis is expected to be better (ABSEy $<$ ABSEx) at all locations inside the vertical cone-shaped regions. This fact, combined with the fact that the experimentally 

significantly larger than ABSEy errors in Tables 1 and 2. One could argue that the accuracy results

437 are 'biased'. However, it should be kept in mind that the goal of this work is to evaluate the 438 localisation performance inside the work zone, i.e., at locations within the driving reach of a moving 439 vehicle where a collision is possible. The geometry of the work zone serves this goal, and 440 consequently affects the statistics of all reported results.

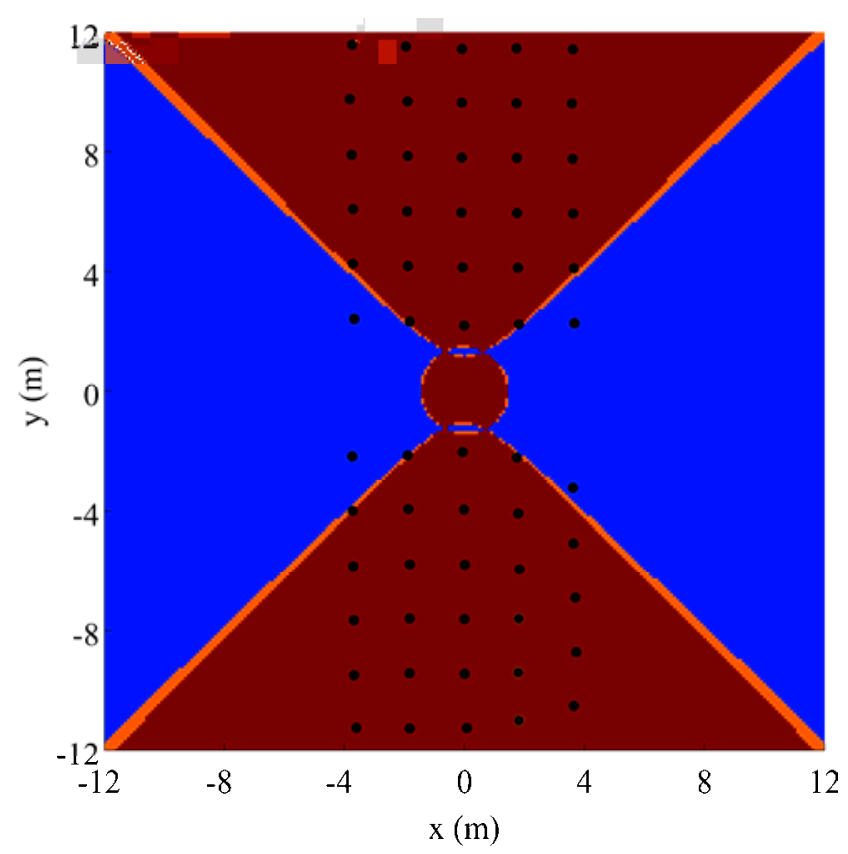

Fig. 10 - Theoretical ratio ABSEx/ABSEy (Eq. 10) calculated over a $24 \times 24 \mathrm{~m}$ region centred at the vehicle. Dots represent actual locations of measurements. Each pixel value encodes whether the ratio ABSEx/ABSEy is greater, less or equal to one. Diagonals and circle around vehicle are loci of points where the ratio is equal to one. ABSEy $<$ ABSEx inside the cone-shaped regions aligned with the yaxis; ABSEx $<$ ABSEy inside the cone-shaped regions aligned with $\mathrm{x}$-axis.

In terms of range, a worker's position estimate is expected to get worse as distances $d_{i}$ increase.

449 Figure 11 shows the DRMS and R95 metrics of all points at increasing distances of the mobile antenna position from the vehicle's frame origin; ground truth is measured with GPSm. As distance grows, more position measurements lie within this distance and more coordinates are included in the 


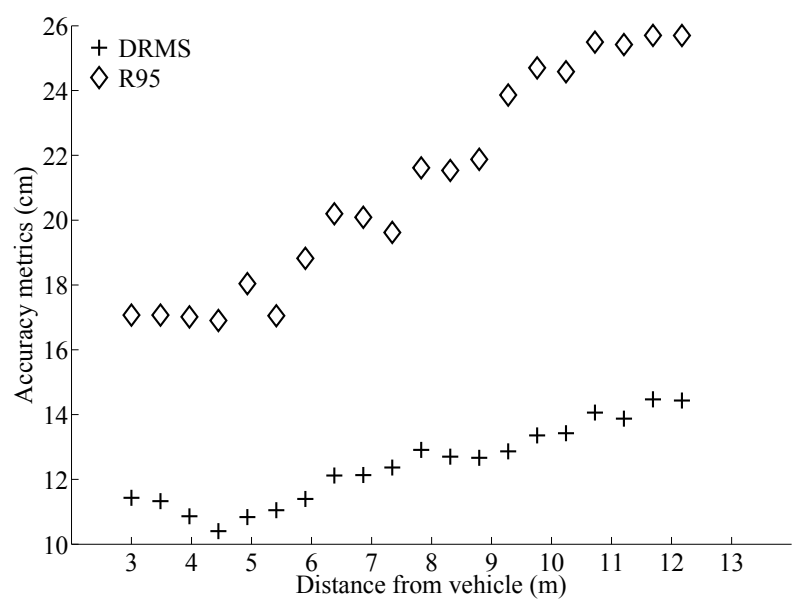

Fig. 11 - DRMS and R95 metrics as functions of distance in open-space static tests.

454 The DRMS metric at $12.2 \mathrm{~m}$ increased by $26.2 \%$ compared to its value at $3 \mathrm{~m}$. The corresponding R95 455 value grew by $50.6 \%$, i.e. twice as much. This means that there is more variance as the antenna moves 456 farther away from the vehicle. To verify this, Fig. 12a and Fig. 12b show the ABSEx and ABSEy 457 metrics respectively (and their standard deviations) of all points, at increasing distances of the 458 measured position from the vehicle's frame origin. From the figures it can be seen that standard 459 deviation of the absolute error along the $x$-axis at $12.2 \mathrm{~m}$ grew by $42.8 \%$ compared to its value at $3 \mathrm{~m}$. 460 The corresponding number along the $y$-axis was $30 \%$.
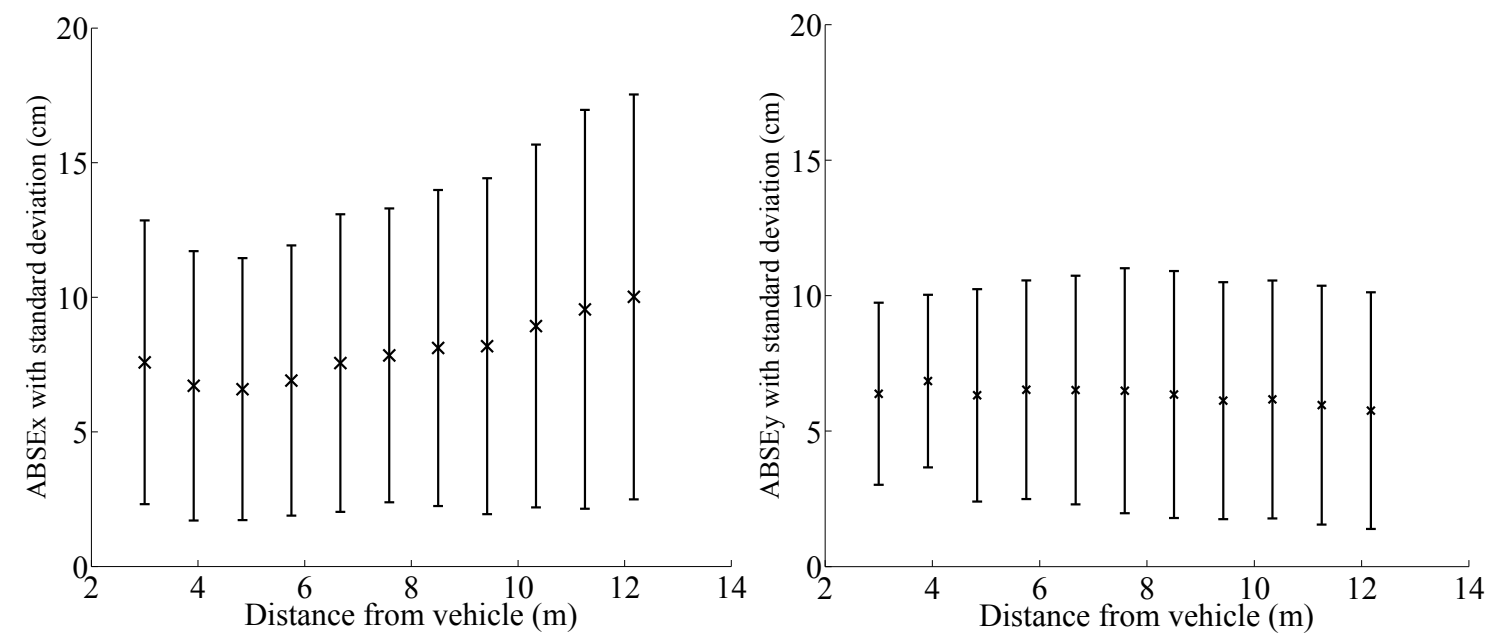
Fig. 12 - (a) ABSEx and (b) ABSEy metrics plotted as a function of distance in open-space static tests.

The above results can be interpreted by looking at the spatial distributions of the theoretically 463 predicted error variances $\operatorname{var}\left(x_{r}\right) / \sigma^{2}$ (Fig. 13a) and $\operatorname{var}\left(y_{r}\right) / \sigma^{2}$ (Fig. 13b), as these can be computed using Eq. (10). Figure 13a shows that ABSEx increases at greater distances along Lines 1 and 2 . In our work zone, the greater the distance from the vehicle, the larger the number of points with large $y$ values that are included in the metric calculation. Hence, ABSEx increases (as shown in Fig. 12a) and results in higher DRMS (and R95) error (as shown in Fig. 11). On the other hand, Fig. 13b shows that ABSEy does not increase significantly within the $|\mathrm{x}| \leq 4 \mathrm{~m}$ zone; this is reflected in the ABSEy metric in Fig. $12 b$.

Finally, the availability computed over all static test points was $100 \%$.
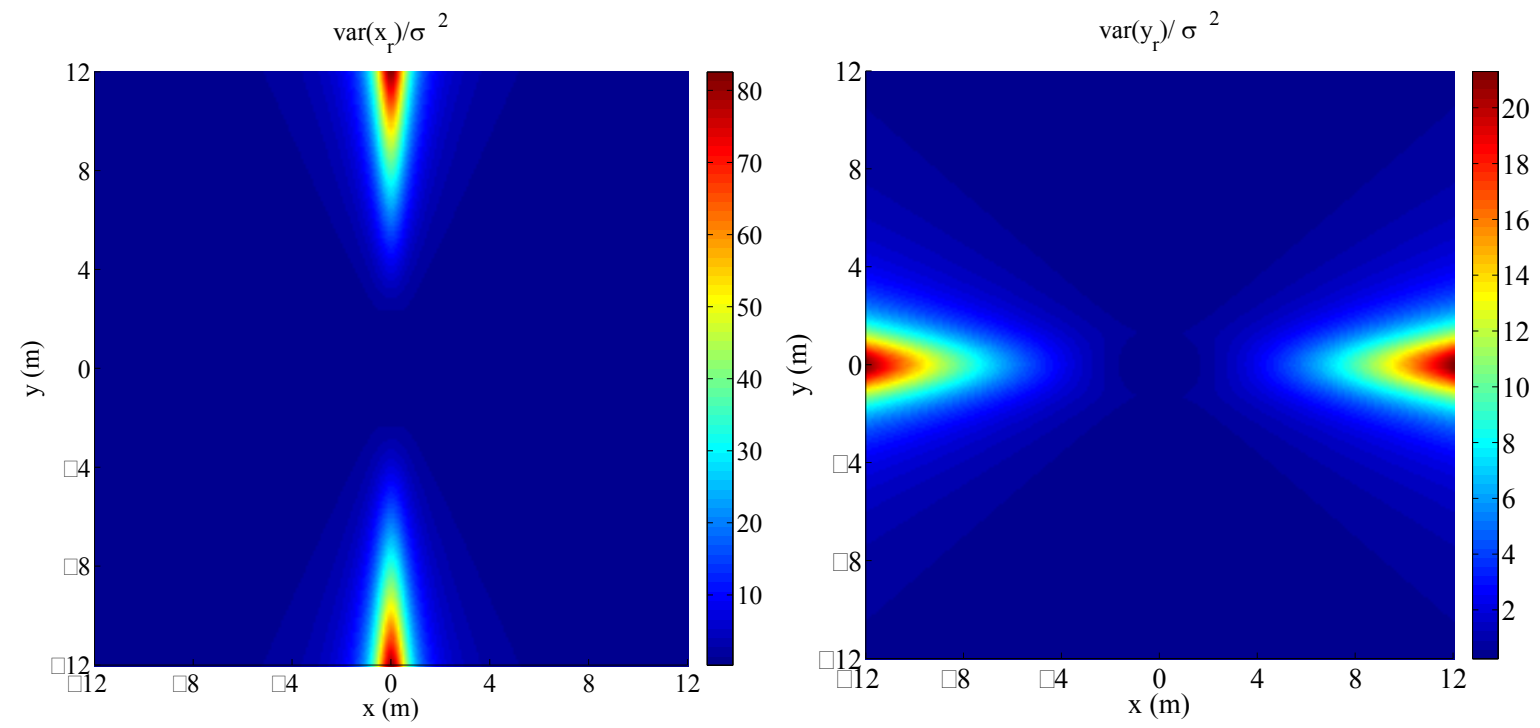

472 Fig. 13 - Spatial distributions of theoretical error variances in open-space static tests: $(\mathrm{a}) \operatorname{var}\left(x_{r}\right) / \sigma^{2}$

473 and (b) $\operatorname{var}\left(\mathrm{y}_{r}\right) / \sigma^{2}$. ABSEx and ABSEy are proportional to the respective variances. 
488 For the dynamic tests the GPSm measurements were used as ground truth, since TS cannot track a 489 moving target. Care was taken to avoid blocking the LOS between the mobile and vehicle beacons. In 490 all data processing for dynamic tests, range data sets $\left(r_{1}, r_{2}, r_{3}, r_{4}\right)$ that contained one or more zeros 491 were not used. Figure 14 shows one repetition (out of ten) of the measured and estimated points on 492 the traversed paths; gaps in the paths indicate missing GPS data or the existence of zero range 493 measurements.

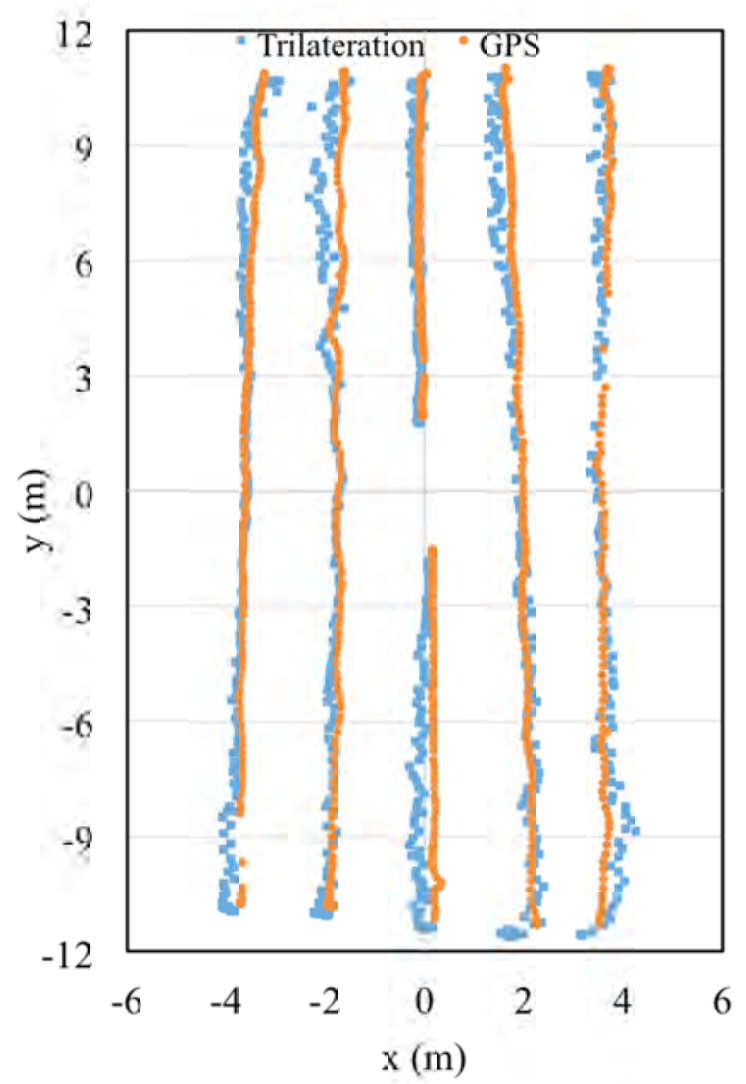

484

487 488 487 488 493 494 495 492
Fig. 14 - Example path during open-space dynamic tests. Dots and squares represent GPSm measurements and trilateration estimates respectively.

Table 3 shows the position accuracy metrics calculated from points on the different paths. The number of valid position measurements varies because of not exactly equal path lengths, and sporadic zero readings. 
491 Table 3 - Open-space dynamic accuracy metrics using GPSm as ground truth (units in $\mathrm{cm}$ ).

492

\begin{tabular}{|c|c|c|c|c|}
\hline & DRMS & $\mathrm{ABSEx} \pm \mathrm{std}$ & $\mathrm{ABSEy} \pm \mathrm{std}$ & R95493 \\
\hline $\begin{array}{l}\text { Line } 1 \\
\text { (1154 points) }\end{array}$ & 22.7 & $14.6 \pm 7.3$ & $7.7 \pm 2.4$ & $\begin{array}{r}43.8^{494} \\
495\end{array}$ \\
\hline $\begin{array}{l}\text { Line } 2 \\
\text { (1756 points) }\end{array}$ & 31.8 & $19.3 \pm 6.1$ & $11.7 \pm 3.4$ & $\begin{array}{r}59.5^{496} \\
497\end{array}$ \\
\hline $\begin{array}{l}\text { Line } 3 \\
\text { (1604 points) }\end{array}$ & 36.5 & $21.3 \pm 6.3$ & $15.2 \pm 3.4$ & $\begin{array}{r}67.2 \\
498\end{array}$ \\
\hline $\begin{array}{l}\text { Front-overall } \\
\text { (2297 points) }\end{array}$ & 32.0 & $17.1 \pm 6.1$ & $15.7 \pm 6.5$ & $51.1_{499}$ \\
\hline $\begin{array}{l}\text { Rear-overall } \\
\text { (2217 points) }\end{array}$ & 31.1 & $20.6 \pm 8.4$ & $8.0 \pm 2.7$ & 65.9500 \\
\hline $\begin{array}{l}\text { All points } \\
\text { (4514 points) }\end{array}$ & 31.6 & $18.8 \pm 7.2$ & $11.9 \pm 4.6$ & $58.1^{501}$ \\
\hline
\end{tabular}

503 The mean absolute errors along the $x$-axis (ABSEx) were larger than the corresponding $y$-axis errors 504 (ABSEy), just like in the static open-space tests. The ABSEx of all the data points was 1.6 times 505 larger than the corresponding ABSEy. The same was true for their variances. A two-sample 506 Kolmogorov-Smirnov test verified that ABSEx and ABSEy do not come from the same distribution 507 (5\% significance).

508 To illustrate the effect of outlier range measurements, the error frequency histogram and the fitted 509 error cumulative density function for the horizontal distance error $e_{x y}$ of all measured points is shown 510 in Fig. 15. To calculate the cumulative density function (cdf) a nonparametric representation of the 511 error probability density function (pdf) was fitted to the data using MATLAB's fitdist() function and 512 the Epanechnikov kernel and the cdf was obtained from this pdf. 


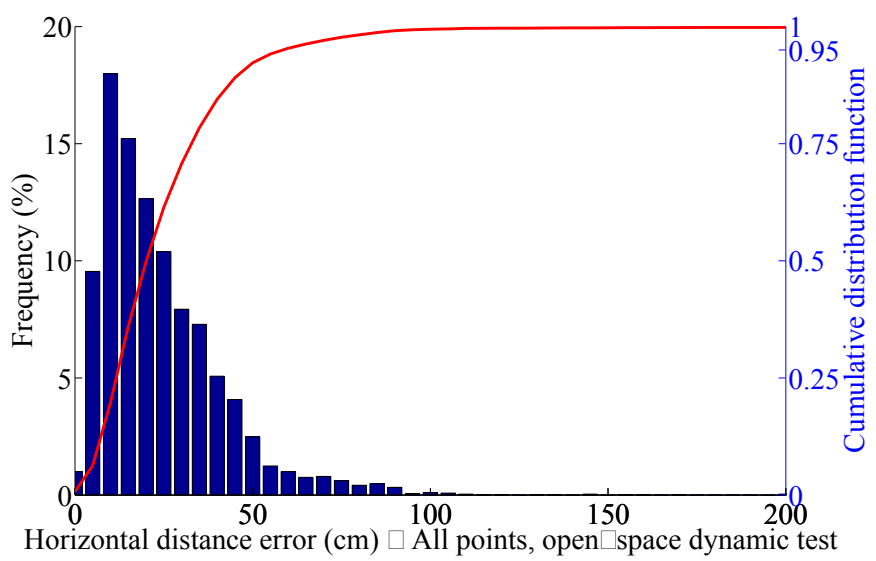

514 Fig. 15 - Open-space dynamic tests: Horizontal distance error histogram and its fitted cumulative 515 probability density function for all points (units in $\mathrm{cm}$ ).

Figure 16 shows the DRMS and R95 metrics as functions of distance of the mobile antenna position 518 from the vehicle frame's origin.

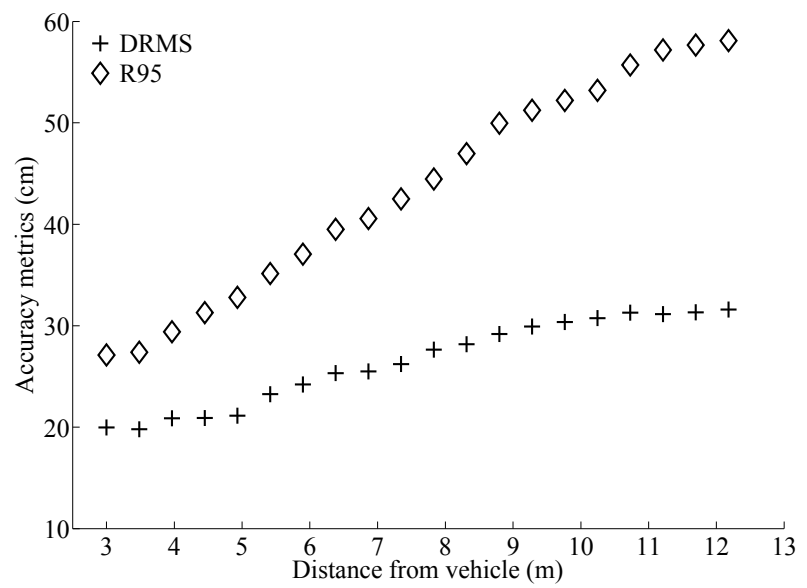

Fig. 16 - DRMS and R95 metrics as functions of distance in open-space dynamic tests.

The DRMS metric at $12.2 \mathrm{~m}$ increased by $58.2 \%$ compared to its value at $3 \mathrm{~m}$. The R95 value between these two distances grew by $114.3 \%$. Clearly, as worker-vehicle distance increases, accuracy suffers and precision worsens because of increased variance and outliers. 
523

524

525

526

527

528

529

530

531

532

533

534

535

536

537

538

539

540

541

542

543

544

545

546

547 Table 4 - Open-space dynamic accuracy metrics with full-LOS body interference using GPSm as

The open-space static and dynamic error results can be compared by considering Fig. 11 and Fig. 16.

At $3 \mathrm{~m}$, the static DRMS and R95 were $11.4 \mathrm{~cm}$ and $17.1 \mathrm{~cm}$ and the respective dynamic metrics were $20.0 \mathrm{~cm}$ and $27.1 \mathrm{~cm}$; these values translate into respective error differences of $75.4 \%$ and $50.6 \%$. At $12.2 \mathrm{~m}$, the static DRMS and R95 were $14.4 \mathrm{~cm}$ and $25.7 \mathrm{~cm}$ and the respective dynamic metrics were $31.6 \mathrm{~cm}$ and $58.1 \mathrm{~cm}$; these values translate into error differences of $119.4 \%$ and $114.3 \%$ respectively. The above numbers indicate that in dynamic trilateration the position error was 1.6 to 2.2 times as large as the static error at the same distance. This is explained primarily by the non-collocation of measurements, i.e., the fact that the range measurements $r_{1}, r_{2}, r_{3}, r_{4}$ in Eq. (5) are available at times $t_{1}, t_{2}, t_{3}, t_{4}$ that are spaced $100 \mathrm{~ms}$ apart; hence they do not correspond to a unique antenna/worker position, but rather to four distinct positions. Therefore, Eq. (5) will result in an estimate that contains two independent sources of error: the dynamic non-collocation error, and the range error $\varepsilon_{i}$ that enters Eq. (5) in both static and dynamic conditions.

Finally, the availability computed over all dynamic open-space test points was $99.5 \%$.

\subsubsection{Human body interference in open space}

The no-LOS and full-LOS experiments were conducted as described in the experimental design section 2.4.4. The position availability for the no-LOS experiments was only $11.1 \%$ and when positions were available the errors were very large. The DRMS over all available position measurements (296 samples) was $819.7 \mathrm{~cm}$ and the R95 was equal to $2495 \mathrm{~cm}$. Clearly, ranging in open space when the human body blocked the LOS completely was unreliable both in terms of accuracy and availability. This is in agreement with results reported by Welch et al. (2002).

The position availability of the full-LOS experiments was $99.7 \%$. The accuracy metrics are given in Table 4.

Table 4 - Open-space dynamic accuracy metries with funl-LOS body interference using GPSm as 
548 ground truth (units in $\mathrm{cm}$ ).

549

\begin{tabular}{|lcc|}
\hline & DRMS & R95 \\
Line 1 & 49.2 & 73.1 \\
(999 points) & & \\
Line 2 & 69.7 & 65.9 \\
(2047 points) & & 62.8 \\
Line 3 & 43.7 & 68.8 \\
(1764 points) & & \\
Front-overall & 45.9 & 68.9 \\
(2634 points) & & \\
Rear-overall & 69.7 & 68.9 \\
(2176 points) & & \\
All points & 57.90 & \\
(4810 points) & & \\
\hline
\end{tabular}

550

551 The fact that DRMS was higher than R95 on Line 2 behind the vehicle led to further analysis of the 552 data. It was found that $0.6 \%$ of the errors were above $200 \mathrm{~cm}$ and that two (out of 4514 ) consecutive 553 position estimates had errors equal to 1745 and $1684 \mathrm{~cm}$ respectively. These two position estimates 554 increased significantly the DRMS without affecting the R95. Removing these two outlier data points 555 reduced the overall DRMS to $46.9 \mathrm{~cm}$; R95 remained essentially the same at $68.5 \mathrm{~cm}$. Compared to 556 the open-space results without body interference in Table 3, the overall DRMS increased significantly 557 (83.2\% including the outliers; $48.7 \%$ without them), whereas the R95 error increased slightly (18.6\%). 558 The presence of the human body in close proximity to the antenna affected negatively the localisation 559 accuracy - but not the availability - in open space when LOS was available.

\subsection{Orchard test results}

\subsubsection{Static tests in the orchard}

562 The points on lines No. 1 (vehicle centreline) and No. 2 under (edges of tree canopies) were measured 563 using TS and GPSm, while the points on line No. 3 (neighbouring row centre-line) were measured 
570 using only GPSm due to lack of unobstructed LOS from the TS. The measurement locations are

571 shown in Fig. 17 for a specific test. The TS measurements are not visible at many locations because

572 they lie below the dots representing the GPSm measurements.

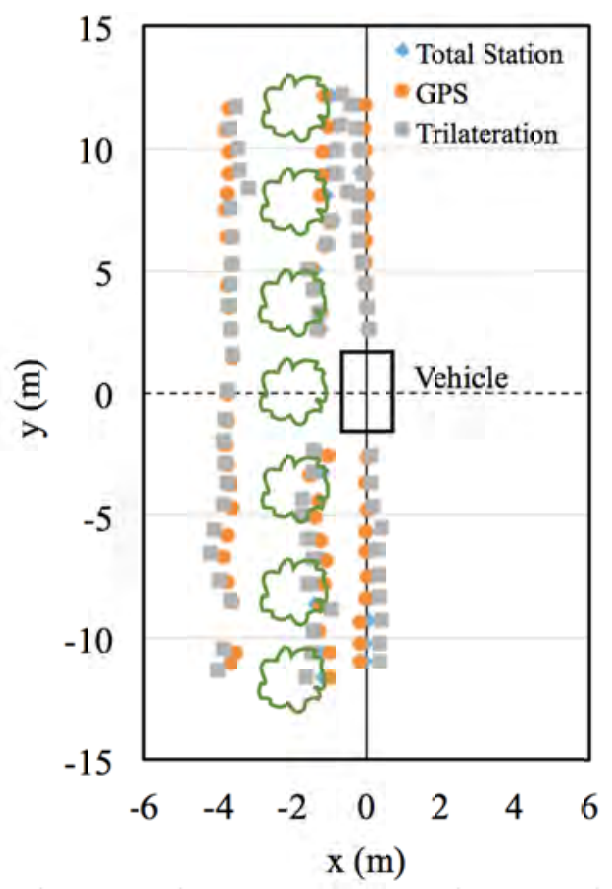

569

574

575

576

573

586

587

588

589

590

591

592

Fig. 17 - Measured and trilaterated positions in the orchard static test during a specific test. Diamonds represent TS measurements; dots represent GPSm measurements; squares represent the average trilateration estimate for each position.

Table 5 contains the accuracy metrics for line 1 and line 2 using TS as ground truth. Overall, when the mobile antenna was under - or partially under - trees (line 2) the trilaterated error was higher than when the antenna was $1 \mathrm{~m}$ away to the right of the tree row (line 1). This was supported by twosample Kolmogorov-Smirnov tests that were applied on the $e_{x}, e_{y},\left|e_{x}\right|,\left|e_{y}\right|$, and $e_{x y}$ error pairs on the two lines; the hypotheses that these errors come from the same distribution were rejected $(5 \%$ significance). The ABSEx errors were significantly larger than the ABSEy errors, as was observed in the open-space experiments. 
Table 5 - Orchard static accuracy metrics using TS as ground truth (units in $\mathrm{cm}$ ).

581

\begin{tabular}{|lcccc|}
\hline & DRMS & ABSEx \pm std & ABSEy \pm std & R95 \\
Line 1 & 20.9 & $15.9 \pm 13.1$ & $3.0 \pm 2.9$ & 41.9 \\
Line 2 & 27.2 & $24.4 \pm 14.9$ & $3.6 \pm 4.1$ & 52.2 \\
All points & 24.4 & $19.3 \pm 14.2$ & $3.3 \pm 3.5$ & 45.8 \\
\hline
\end{tabular}

582

583 Figure 18 shows the DRMS and R95 metrics for all points on Lines 1 and 2 combined, as functions of 584 distance of the mobile antenna position from the vehicle's frame origin; ground truth was measured 585 with TS.

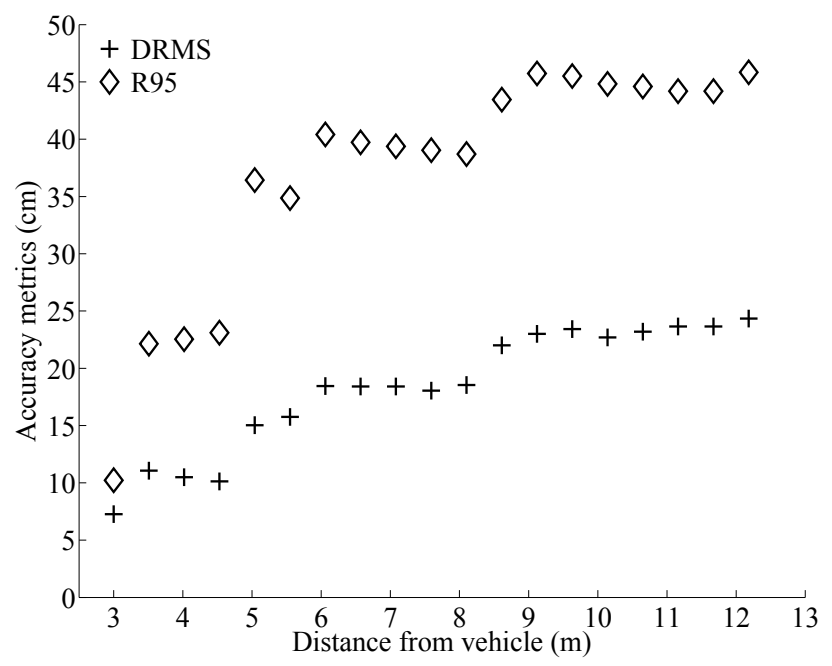

Fig. 18 - DRMS and R95 metrics as functions of distance in orchard static tests.

The DRMS error at $12.2 \mathrm{~m}$ was $24.3 \mathrm{~cm}$ and increased by $235 \%$ compared to its value at $3 \mathrm{~m}$; the corresponding R95 value was $45.8 \mathrm{~cm}$ and increased by 349\%. A comparison between Fig. 11 and Fig. 18 for the static tests reveals that the orchard static DRMS is comparable to the open-space DRMS when the antenna is close to the vehicle, and that it increases faster as distance increases,

591 becoming almost twice as large as the open-space DRMS at $12.2 \mathrm{~m}$. A similar trend is present for the R95 accuracy metric.

593 The results shown in Table 4 and Fig. 18 suggest that the presence of trees - especially in LOS - 
594

595

596

597

598

599

600

601

602

603

604

605

606

607

608

609

610

611

612

613

614

615

616

617

618

affects negatively the positioning accuracy of the trilateration system. An explanation is that as distance increases, more foliage enters in the propagation paths of the radio signals of the four vehicle beacons, and the resulting increased scattering and multipath introduce larger errors $\varepsilon_{i}$, which can be significantly different for each beacon (Al-Nuaimi \& Hammoudeh, 1994). These errors enter into Eq. (5) and degrade the trilateration position estimate.

In order to assess the trilateration accuracy between tree rows and to compare static and dynamic accuracies, RTK GPS data must be used as "ground truth", since the TS laser beam can neither "see" the mobile antenna on line 3 or track the moving antenna on any path. However, it is known that RTK GPS accuracy and precision degrade when the antenna is under - or close to - tree canopies because of reduced signal strength and multipath/scattering effects while the electromagnetic wave is penetrating the branches and canopies of trees. Various researchers (e.g., Lee an Ge, 2006; Brach \& Zasada, 2014) have reported RTK GPS DRMS static errors in the order of $10-40 \mathrm{~cm}$ or more, when the GPS antenna is near or under trees, using TS as ground truth. In order to compare TS and GPSm measurements in our orchard, the DRMS and R95 metrics were computed independently for Line 1 and Line 2. TS measurements were treated as "absolute ground truth", since they are not affected by foliage. On Line 1, DRMS and R95 were $11.8 \mathrm{~cm}$ and $20.9 \mathrm{~cm}$ respectively. The corresponding values were $1.8 \mathrm{~cm}$ and $3.2 \mathrm{~cm}$ in the open-space tests. Clearly, the presence of the trees affected RTK GPS accuracy of positions on line 1, although the measurement points were not under the tree canopies. Points on line 2, which were partially under the tree canopies, had higher DRMS and R95 values than line 1 , equal to $14.1 \mathrm{~cm}$ and 24.1 respectively.

Obviously, the GPSm cannot be used to provide accurate measurements of the true antenna positions, like in the open-space experiments, because it adds a significant - independent - error to the measured position. Keeping in mind that DRMS and R95 correspond to radii of circles around the true position, GPSm measurements can be used to provide upper bounds of the trilaterated errors. These bounds are simply the sums of two radii around the true position, i.e., the trilaterated-versus-GPSm error metrics 
619

620

621

622

623

624

625

626

627

628

629

630

\begin{tabular}{|lcc|}
\hline & DRMS upper bound & R95 upper bound \\
Line 1 & $42.1(30.3+11.8)$ & $78.0(57.1+20.9)$ \\
Line 3 & $36.5(24.7+11.8)$ & $71.9(51.0+20.9)$ \\
All points & $39.3(27.5+11.8)$ & $78.1(57.2+20.9)$ \\
\hline
\end{tabular}

and the GPSm-versus-TS position error metrics $(11.8 \mathrm{~cm}$ and $20.9 \mathrm{~cm}$ respectively). is $20.9 \mathrm{~cm})$. GPSm-versus-TS position error (right term in parenthesis).
631

632

633

634

635

636

637

638

639

640

641

Table 6 presents the trilaterated-versus-GPSm accuracy bounds for line 1 and line 3 . The calculated error bounds are conservative and sometimes can be much larger than the actual errors. For example, a comparison between the error metrics along Line 1 from Table 4 (ground truth TS; first row) and Table 5 (ground truth GPSm; first row) reveals that in these tests the upper bounds were approximately twice as large as the measured values (e.g., DRMS bound is $42.1 \mathrm{~cm}$, whereas DRMS

Table 6 - Static position error bounds in an orchard using GPSm as ground truth (units in $\mathrm{cm}$ ). Each bound value is the sum of two errors: trilaterated-versus-GPSm error (left term in parenthesis) and

In the orchard, static testing availability was $100 \%$ and the tree trunks and low-height foliage in this orchard did not block radio communication among the beacons within the tested range.

\subsubsection{Dynamic tests in the orchard}

During dynamic testing, a human carried the mobile radio and GPSm while walking on the paths specified by the experimental design and staying away from the antennas' LOS and from the mobile antenna itself. The GPSm was used as ground truth. Fig. 19 shows a sample path. The corresponding errors for line 1 and line 3 are given in Table 7. Error data for points on line 2 are not available due to foliage blocking the GPSm signal. As with the open-space dynamic tests, statistics were calculated based on varying sample size because of different path lengths and sporadic zero readings. 


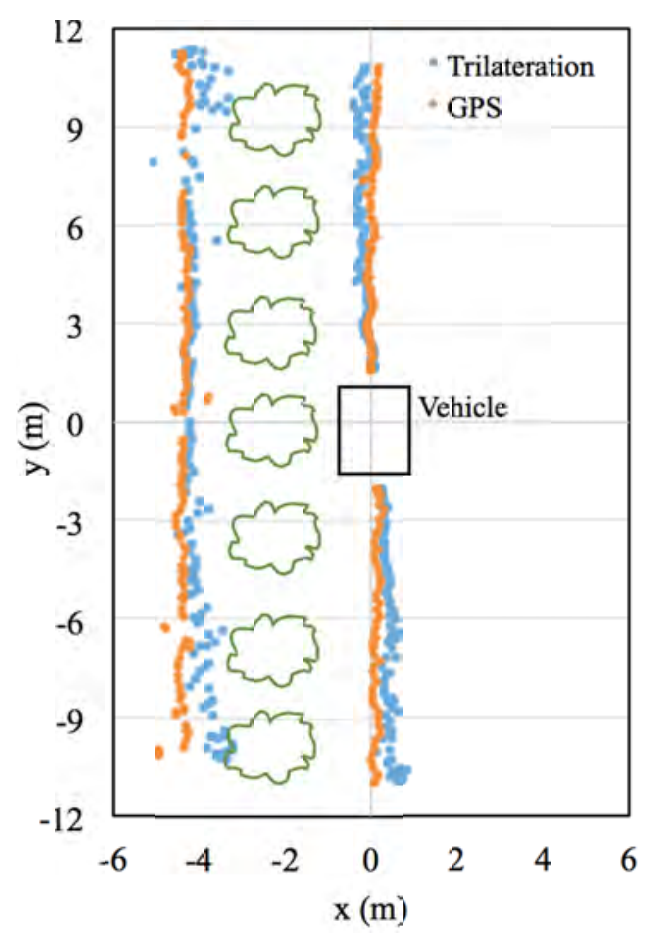

644

647

648

647

652

653

654

651

Fig. 19 - Points on a sample path during dynamic testing in the orchard without body interference. Dots and squares represent GPSm measurements and trilateration estimates respectively.

Table 7. Dynamic position error bounds in an orchard using GPSm as ground truth (units in $\mathrm{cm}$ ). Each bound value is the sum of two errors: trilaterated-versus-GPSm error (left term in parenthesis) and GPSm-versus-TS position error (right term in parenthesis).

\begin{tabular}{|lcc|}
\hline & DRMS upper bound & R95 upper bound \\
Line 1 & $44.8(33.0+11.8)$ & $83.1(62.2+20.9)$ \\
$(1164$ points $)$ & $76.6(64.8+11.8)$ & $147.0(126.1+20.9)$ \\
Line 3 & & \\
(947 points) & $61.6(49.8+11.8)$ & $116.7(95.8+20.9)$ \\
All points & & \\
$(2111$ points $)$ & & \\
\hline
\end{tabular}

A direct comparison of Tables 6 and 7 reveals a small increase in the upper bounds of the DRMS and R95 positioning errors on line 1, and a dramatic increase (doubling) of these error bounds along Line 3, which lies in the middle between two tree rows on the left side of the vehicle. A statistical twosample Kolmogorov-Smirnov test verified that the errors did not follow the same distribution. This can be attributed to the combined effects of the dynamic non-collocation error in the range measurements (moving antenna) and the multipath error due to signal scattering and reflection in the 
657

658

659

660

661

662

663

664

665

666

667

668

669

670

671

672

presence of tree foliage. Finally, position availability during dynamic testing was $97.5 \%$.

\subsubsection{Human body interference in the orchard}

The experiments for human body interference in the orchard were conducted as described in the experimental design section 2.4.4. Improved GPS conditions enabled measurements at the periphery of the tree canopies (line 2 of Fig. 8b). Figure 20 shows the paths traversed during one repetition (out of ten) of the full-LOS experiments.

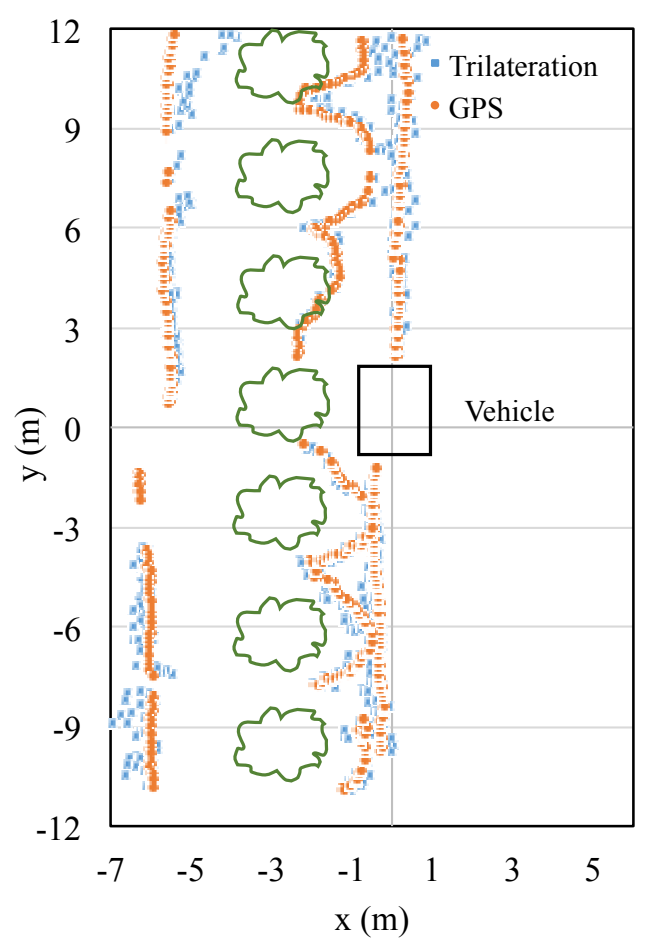

Fig. 20 - Points on a sample path during dynamic testing in the orchard with full-LOS and body interference. Dots and squares represent GPSm measurements and trilateration estimates respectively.

The position availability of the full-LOS experiment was $99.3 \%$. The availability of the no-LOS experiment was $60.2 \%$; much lower than the full-LOS availability, but significantly higher than the measured open-space availability (11.1\%). This increase can be attributed to the presence of trunks and branches, which seem to have allowed for multi-path propagation. This finding is in agreement with published results (Welch et al., 2002). 
680

\begin{tabular}{|lcc|}
\hline & DRMS upper bound & R95 upper bound \\
Line 1 & $59.2(47.4+11.8)$ & $82.5(61.6+20.9)$ \\
$(1709$ points $)$ & $56.1(44.3+11.8)$ & $86.0(65.1+20.9)$ \\
Line 2 & & \\
$(1903$ points $)$ & $67.1(55.3+11.8)$ & $135.2(114.3+20.9)$ \\
Line 3 & & \\
$(1754$ points $)$ & $63.4(51.6+11.8)$ & $108.0(87.1+20.9)$ \\
All points (Lines $1+3)$ & & \\
(5366 points) & & \\
\hline
\end{tabular}
parenthesis) and GPSm-versus-TS position error (right term in parenthesis).
681

682

686

Table 8 - Upper bounds of position errors in full-LOS body interference experiments in the orchard (units in $\mathrm{cm}$ ). Each bound value is the sum of two errors: trilaterated-versus-GPSm error (left term in

Table 9 - Upper bounds of position errors in no-LOS body interference experiments in the orchard (units in $\mathrm{cm}$ ). Each bound value is the sum of two errors: trilaterated-versus-GPSm error (left term in parenthesis) and GPSm-versus-TS position error (right term in parenthesis).

\begin{tabular}{|lcc|}
\hline & $\begin{array}{c}\text { DRMS upper bound } \\
\text { Line 1 }\end{array}$ & R95 upper bound \\
$(645$ points $)$ & $138.7(126.9+11.8)$ & $278.9(258.0+20.9)$ \\
Line 2 & $105.2(93.4+11.8)$ & $150.7(129.8+20.9)$ \\
$(738$ points $)$ & & \\
Line 3 & $127.6(115.8+11.8)$ & $181.5(160.6+20.9)$ \\
$(487$ points $)$ & & \\
All points (Lines 1+3) & $123.6(111.8+11.8)$ & $217.8(196.9+20.9)$ \\
$(1870$ points $)$ & & \\
\hline
\end{tabular}


experiments without antenna-body proximity (Table 7) and the full-LOS experiments with the body in close proximity to the antenna (Table 8) showed that the errors do not follow the same distribution. The upper bound of the localisation error along line 1 increased from $44.8 \mathrm{~cm}$ to $59.2 \mathrm{~cm}$. Given that tree interference is minimal along this line, this increase can be attributed to the proximity of the body. On line 3 where the trees and body form a dense multi-path environment, the proximity of the body seems to slightly reduce the error. Overall, the errors were comparable and body proximity did not seem to increase errors significantly. The no-LOS experiment resulted in errors that were typically twice as large as the full-LOS errors. This can be explained by the fact that radio communication was made possible mostly via multipath propagation, as attested by the increased availability in the orchard compared to that of open space.

\section{Conclusions}

An ultra-wideband radio-ranging and trilateration system was developed to localise workers in orchards. Trilateration was performed based on four range estimates between a vehicle's beacon antennas and the antenna of a mobile radio unit, which was carried by a human. The system's position-estimation accuracy and availability were assessed at locations close to the vehicle in a variety of conditions. In the absence of a human close to the mobile antenna, localisation was tested when the antenna was static and when it was moving, both in open space and in an orchard. When a human carried the antenna on a belt (close proximity) and walked, localisation was tested in open space and in an orchard, with full and zero LOS availability. The major conclusions of this study can be summarised as follows.

\subsection{Effects of vehicle beacon configuration}

In the open-space static and dynamic tests, and in the orchard static tests, the mean absolute error along the vehicle's heading (ABSEy) ranged from $3.3 \mathrm{~cm}$ to $11.9 \mathrm{~cm}$, and was 1.6 to 5.8 times smaller 
714

715

716

717

718

719

720

721

722

723

724

725

726

727

728

729

730

731

732

733

734

735

736

737

738

than the error along the axis perpendicular to the vehicle (ABSEx). Also, although accuracy decreased with increasing distance (Fig. 11), the accuracy along the vehicle's axis (ABSEy) did not change significantly within the $|\mathrm{x}| \leq 4 \mathrm{~m}$ work zone (Fig. 12b). This was due to the geometrical configuration of the vehicle beacons, and the geometry of the work zone (Figs. 10, 13a, 13b). The fact that ABSEy was smaller than ABSEx is favourable, because accurate worker localisation in the vehicle's direction of motion may enable more effective braking decisions.

\subsection{Effects of moving (vs. static) antenna without antenna-body proximity.}

When the antenna was moving, the accuracy of the trilateration system was worse than in static conditions. In open space and in the orchard, the DRMS and R95 position errors at walking speed were 1.6 to 2.2 times larger than the corresponding errors at the same distance under static conditions (Tables 2, 3, 6, 7). This is explained primarily by the non-collocation of measurements, i.e., the fact that the four ranges used for trilateration do not correspond to the same position. The non-collocation error could be lowered if range measurements were not filtered in real time, thus increasing the beacons' polling rate. Such an approach would increase the range error variance, so its benefits must be examined in real conditions. Given that vehicles and workers in orchards do not move at high speeds, this error is not expected to prohibit the use of active beacons for trilateration.

\subsection{Effects of presence of trees without antenna-body proximity}

The accuracy of the trilateration system in the orchard was worse than in open space. The position error in the orchard was higher under both static (Table 2, DRMS $14.4 \mathrm{~cm}$ vs. Table 6 DRMS 39.3 $\mathrm{cm}$ ) and dynamic conditions (Table 3, DRMS $31.6 \mathrm{~cm}$ vs. Table 7, DRMS $61.6 \mathrm{~cm}$ ). This can be attributed to multipath error due to signal scattering and reflection from tree trunks and foliage. Closer to the vehicle, the DRMS and R95 errors in the orchard were comparable to the open-space errors, but grew faster than open-space errors as distance increased; at a distance of approximately 12 m they were almost twice as large (Figs. 11, 18). Overall, the trees in the orchard did not seem to 
block radio communication among the beacons at the tested distances $(<12.2 \mathrm{~m})$. In open space the availability over all test points was $100 \%$ and $99.5 \%$ for static and dynamic tests respectively. In the orchard, static testing availability was $100 \%$ and during dynamic testing it dropped to $97.5 \%$. It is expected that some predictive filtering could compensate for short-time data outages.

\subsection{Combined effects of moving antenna and tree-presence without antenna-body proximity}

In the dynamic experiments in the orchard, when the antenna was carried between tree rows to the side of the vehicle (line 3, Tables 6,7) the bounds of the DRMS and R95 position errors were almost twice as high as the corresponding errors on line 1. Given that a row of trees stood between the vehicle and the moving antenna, this large increase in error could be attributed to the combined effects of the dynamic non-collocation error and the foliage-induced multipath error. However, positioning accuracy is not critical when a worker works in a different row than the vehicle because a collision with the worker is not possible.

\subsection{Effects of antenna-body proximity with antenna moving at walking speed}

When the human body blocked completely the signal propagation among antennas (no-LOS), trilateration in open space was completely unreliable, both in terms of accuracy and availability. Under the same no-LOS conditions in the orchard, the availability increased more than five times, due to multi-path propagation; however, the accuracy was still extremely poor (Table 9). This is a very important shortcoming, because the proposed system relies on radio antennas carried/worn by workers. A possible solution would be to use two antennas on opposite sides of the body (e.g., leftand-right on a belt), so that full-LOS is available most of the time (P400 RCM supports two antennas). Another - perhaps complementary - approach is to use a filter (e.g., Kalman) to calculate worker positions during short-time data outages. 
763 Under full-LOS conditions, the localisation accuracy over all points in open space decreased

764 significantly compared to that without antenna-body proximity (Tables 3, DRMS $31.6 \mathrm{~cm}$ vs. Table 4, 765 DRMS 57.9). However, under full-LOS in the orchard, the accuracy and availability were similar with 766 and without antenna-body proximity (Table 7, DRMS $61.6 \mathrm{~cm}$ vs. Table 8, DRMS $63.4 \mathrm{~cm}$ ). This 767 finding is very promising for localisation in orchard settings and could be attributed to the multi-path 768 environment created by the combination of trunks, branches, leaves and the human body, which 769 allowed for multi-path signal propagation. Also, the position availability with full-LOS was very high $77099.3 \%$ and indicated that the proximity of the worker's body was not significant.

771

772 In overall conclusion, active radio ranging and trilateration seems to offer a workable approach for 773 human detection and localisation by vehicles moving slowly in orchards. Of course, each worker 774 would need to carry an active beacon. However, the system would not rely on cameras, laser scanners 775 and advanced perception algorithms, although it could be used in addition to them as a 776 complementary sensing modality. Issues related to sporadic position unavailability and outlier errors 777 (R95 much higher than DRMS) could be addressed by suitable filtering. As the need for autonomous 778 vehicles in open-field and horticultural crops increases, safe navigation systems that detect and 779 localise human workers in compliance with - yet to be developed - safety standards are expected to 780 become crucial for agricultural robotic vehicle adoption and deployment.

\section{Acknowledgements}

782 We would like to thank the anonymous reviewers for their constructive comments. This work was 783 funded by NIFA-USDA Award 2013-67021-21073 and NIFA-USDA Hatch Multi-State Grant $784 \quad 1001069$. 


\section{References}

Al-Nuaimi, M.O., and Hammoudeh, A.M. (1994). Measurements and predictions of attenuation and scatter of microwave signals by trees. IEE Proceedings Antennas Propagation, 141: 70-76.

Bharadwaj, R., Parini, C. and Alomainy, A. (2015). Experimental Investigation of 3-D Human Body Localization Using Wearable Ultra-Wideband Antennas. IEEE Transactions on Antennas and Propagation, 63(11), 5035-5044.

Barawid, O. C., Mizushima, A., Ishii, K., and Noguchi, N. (2007). Development of an autonomous navigation system using a two-dimensional laser scanner in an orchard application. Biosystems Engineering, 96(2), 139-149.

Bergerman, M., Singh, S., and Hamner, B. (2012). Results with autonomous vehicles operating in specialty crops. 2012 IEEE International Conference on Robotics and Automation. Saint Paul, Minnesota, May 14-18 2012.

Bull, M. (2011). WSU scientists perfecting mechanical harvest of sweet cherries. Available at http://researchnews.wsu.edu/physical/143.html Accessed on 15.5.2015.

Bellotto, N., and Hu, H. (2009). Multisensor-based human detection and tracking for mobile srvice robots. IEEE Transactions on Systems, Man, and Cybernetics, Part B: Cybernetics. 39(1), 167181.

Bochtis, D., Griepentrog, H.W., Vougioukas, S., Busato, P., Berruto, R., Zhou, K. (2015). Route planning for orchard operations, Computers and Electronics in Agriculture, 113, 51-60.

Brach, M., Zasada, M. (2014). The Effect of Mounting Height on GNSS Receiver Positioning Accuracy in Forest Conditions. Croatian Journal of Forest Engineering, 35(2), 245-253.

Chang, S., Mitsumoto, N., and Burdick, J.W. (2009). An algorithm for UWB radar-based human detection. IEEE Radar Conference. May 4-8, 2009. Pasadena, CA. pp: 1-6. 
809 Chehri, A., Fortier, P., and Tardif, P. M. (2009). UWB-based sensor networks for localization in 810 mining environments. Ad Hoc Networks. 7(5): 987-1000.

811 Cheng, T., Venugopal, M., Teizer, J., and Vela, P. A. (2011). Performance evaluation of ultra 812

Dollar, P., Wojek, C., Schiele, B., \& Perona, P. (2012). Pedestrian detection: An evaluation of the Automation in Construction. 20(8): 1173-1184. state of the art. IEEE Transactions on Pattern Analysis and Machine Intelligence, 34(4), 743-761.

Freitas, G., Hamner, B., Bergerman, M., and S. Singh. (2012). A practical obstacle detection system for autonomous orchard vehicles. 2012 IEEE/RSJ International Conference on Intelligent Robots and Systems. October 7-12, 2012. Vilamoura, Algarve, Portugal.

Gandhi, T., Trivedi, M., (2007). Pedestrian protection systems: Issues, survey, and Challenges. IEEE Transactions on Intelligent Transportation Systems 8 (3), 413-430.

He, L., Arikapudi, R., Khosro Anjom, F., and Vougioukas, S. (2014). Worker position tracking for safe navigation of autonomous orchard vehicles using active ranging. ASABE paper No. 141913710 St. Joseph, Mich.: ASABE.

Hertz, T., and Zahniser, S. (2013). Is there a farm labor shortage? American Journal of Agricultural Economics. 95(2): 476-481.

ISO 10218-1:2011 Robots and robotic devices - Safety requirements for industrial robots - Part 1: Robots. International Organization for Standardization, http://www.iso.org.

ISO/DTS 15066: 2014 Robots and robotic devices - Safety requirements for industrial robots Collaborative operation. International Organization for Standardization, http://www.iso.org.

Khoury, H. M., and Kamat, V. R. (2009). Evaluation of position tracking technologies for user localization in indoor construction environments. Automation in Construction. 18(4): 444-457. 
832 Kohanbash, D., Bergerman, M., K. Lewis, and Moorehead, S. (2012). A Safety Architecture for Autonomous Agricultural Vehicles. ASABE paper No. 121337110 St. Joseph, Mich.: ASABE.

Lee, I. S., and Ge, L. (2006). The performance of RTK-GPS for surveying under challenging environmental conditions. Earth, planets and space, 58(5), 515-522.

Leone, F., Nottingham, R., Nelson, L. (1961). The Folded Normal Distribution. Technometrics, 3(4): $543-550$.

Linker, R., and Blass, T. (2008). Path-planning algorithm for vehicles operating in orchards. Biosystems engineering, 101(2), 152-160.

Mabrouk, B., Talbi, L., Nedil, M., and Hettak, K. (2012). The Effect of the Human Body on MIMOUWB Signal Propagation in an Underground Mine Gallery, Journal of Electromagnetic Waves and Applications, 26(4), 560-569.

Manolakis, D.E. (1996). Efficient solution and performance analysis of 3-D position estimation by trilateration. IEEE Transactions on Aerospace Electronic Systems, 32(4), 1239-1248.

Moorehead, S. J., Wellington, C. K., Gilmore, B. J., and Vallespi, C. (2012). Automating orchards: A system of autonomous tractors for orchard maintenance. IEEE/RSJ Int. Conf. on Intelligent Robots and Systems (IROS) Workshop on Agricultural Robotics, 2012.

Navidi, W., Murphy Jr. W. S., Hereman, W. (1998). Statistical methods in surveying by trilateration. Comput. Stat. Data Anal. 27, 209-217.

Niemeier, W., Johannes, L. Tengen, D. (2014). Local Positioning Solution to Ensure Workers Safety behind Construction Vehicles. In Proceedings of the 4th International Conference on Machine Control and Guidance, Braunschweig, Germany, 19-20 March, 2014. Available online: http://www.digibib.tu-bs.de/?docid=00056119 (accessed on 20/5/2014).

Porto, S.M.C., Arcidiacono, C., Giummarra, A., Anguzza, U., \& Cascone, G. (2014). Localisation and identification performances of a real-time location system based on ultra wide band technology 
856

857

858

859

860

861

862

863

864

865

866

867

868

869

870

871

872

873

874

875

876

877

878

for monitoring and tracking dairy cow behaviour in a semi-open free-stall barn. Computers and Electronics in Agriculture, 108, 221-229.

Sunderman, C., and Waynert, J. (2012). An overview of underground coal miner electronic tracking system technologies. In Industry Applications Society Annual Meeting (IAS), 2012 IEEE, pp: 1-5.

Vougioukas, S., Anastassiu, H.T., Regen, C., Zude, M. (2013). Influence of foliage on radio path losses (PLs) for wireless sensor network (WSN) planning in orchards. Biosystems Engineering, 114(4), 454-465.

Welch, T.B., Musselman, R.L., Emessiene, B., Gift, P.D., Choudhury, D.K., Cassadine, D.N. and Yano, S.M. (2002). The effects of the human body on UWB signal propagation in an indoor environment. IEEE Journal on Selected Areas in Communications, 20(9), 1778-1782.

Ye, Y., He, L., Zhou, J., Zhang, Q., and Lewis, K. (2013). A remote controlled bin-dog for transferring bins in orchard environments. ASABE paper No. 131596530 St. Joseph, Mich.: ASABE.

Zhang, Q., Feid, J., and Noguchi, N. (1999). Agricultural vehicle navigation using multiple guidance sensors. Proceedings of the International Conference on Field and Service Robotics. pp: 293298.

Zhou, Y. (2011). A closed-form algorithm for the least-squares trilateration problem. Robotica, 29(03), 375-389.

Fig. 1 - The UWB localisation system consists of: 1) Utility vehicle, 2) Four vehicle radio beacons, 3) Mobile radio beacon, 4) Mobile RTK GPS, 5) Vehicle RTK GPS, and 6) Total Station.

Fig. 2 - Radio unit A measures and reports the distance (red dotted line) between the center points of antennas $\mathrm{A}$ and $\mathrm{B}$. 
Fig. 3 - Coordinate frames of the vehicle, radio beacons, GPSv, GPSm, and TS.

Fig. 4 - The illustration of four ranges from vehicle beacons to the mobile beacon.

Fig. 5 - Static test design in open space; black dots represent the locations of the static test points.

Fig. 6 - Image from the pear tree orchard used for localization experiments.

Fig. 7 - Static test design in orchard environment; black dots represent the locations of the static test

884 points.

Fig. 8 - Dynamic test paths in: (a) open space, and (b) in an orchard.

886

887

888

889

890

891

892

893

894

895

896

Fig. 12 - (a) ABSEx and (b) ABSEy metrics plotted as a function of distance in open-space static tests.

Fig. 9 - Open-space static test: positions measured with TS and GPSm and estimated via trilateration in a specific test. Diamonds represent TS measurements; dots represent GPSm measurements; squares represent the average trilateration estimate for each position.

Fig. 10 - Theoretical ratio ABSEx/ABSEy (Eq. 10) calculated over a 24 x $24 \mathrm{~m}^{2}$ square region centered at the vehicle. Dots represent actual locations of measurements. Each pixel value encodes whether the ratio ABSEx/ABSEy is greater, less or equal to one. Diagonals and circle around vehicle are loci of points where the ratio is equal to one. ABSEy $<$ ABSEx inside the cone-shaped regions aligned with the $y$-axis; ABSEx $<$ ABSEy inside the cone-shaped regions aligned with $x$-axis.

Fig. 11 - DRMS and R95 metrics as functions of distance in open-space static tests.

Fig. 13 - Spatial distributions of theoretical error variances in open-space static tests: (a) $\operatorname{var}\left(x_{r}\right) / \sigma^{2}$ and (b) $\operatorname{var}\left(\mathrm{y}_{r}\right) / \sigma^{2}$. ABSEx and ABSEy are proportional to the respective variances.

Fig. 14 - Example path during open-space dynamic tests. Dots and squares represent GPSm 
measurements and trilateration estimates respectively.

903

904 Fig. 15 - Open-space dynamic tests: Horizontal distance error histogram and its fitted cumulative 905 probability density function for all points (units in $\mathrm{cm}$ ).

Fig. 16 - DRMS and R95 metrics as functions of distance in open-space dynamic tests.

906 Fig. 17 - Measured and trilaterated positions in the orchard static test during a specific test. Diamonds

907 represent TS measurements; dots represent GPSm measurements; squares represent the average 908 trilateration estimate for each position.

Fig. 18 - DRMS and R95 metrics as functions of distance in orchard static tests.

909 Fig. 19 - Points on a sample path during dynamic testing in the orchard without body interference. 910 Dots and squares represent GPSm measurements and trilateration estimates respectively.

Fig. 20 - Points on a sample path during dynamic testing in the orchard with full-LOS and body 913 interference. Dots and squares represent GPSm measurements and trilateration estimates respectively. 